\title{
Ultra-luminous Infrared galaxies in Sloan Digital Sky Survey Data Release 6
}

\author{
L. G. Hou ${ }^{1}$, Xue-Bing $\mathrm{Wu}^{2}$, J. L. $\operatorname{Han}^{1}$
}

\begin{abstract}
Ultra-luminous infrared galaxies (ULIRGs) are interesting objects with dramatic properties. Many efforts have been made to understand the physics of their luminous infrared emission and evolutionary stages. However, a large ULIRG sample is still needed to study the properties of their central black holes (BHs), the $\mathrm{BH}$-host galaxy relation, and their evolution. We identified 308 ULIRGs from the Sloan Digital Sky Survey Data Release 6, and classified them into the NL ULIRGs (with only narrow emission lines) and the Type I ULIRGs (with broad emission lines). About $56 \%$ of ULIRGs in our total sample show interaction features, and this percentage is $79 \%$ for redshift $z<0.2$. Optical identifications of these ULIRGs show that the active galactic nucleus percentage is at least $49 \%$, and the percentage increases with the infrared luminosity. We found 62 Type I ULIRGs, and estimated their BH masses and velocity dispersions from their optical spectra. Together with known Type I ULIRGs in the literature, a sample of 90 Type I ULIRGs enables us to make a statistical study. We found that the BH masses of Type I ULIRGs are typically smaller than those of Palomar-Green (PG QSOs), and most Type I ULIRGs follow the $M_{\mathrm{BH}}-\sigma$ relation. However, some ULIRGs with a larger Eddington ratio deviate from this relation, even though the line width of the $\left[\mathrm{O}_{\mathrm{III}}\right]$ narrow-line $(\mathrm{NL})$ core or the $\left[\mathrm{S}_{\mathrm{II}}\right]$ line was used as the surrogate of velocity dispersion. This implies that at least some ULIRGs are probably still in the early evolution stage toward QSOs. The anti-correlation between the mass deviation from the $M_{\mathrm{BH}}-\sigma$ relation and the Eddington ratio supports that the evolution of Type I ULIRGs is probably followed by the building up of the $M_{\mathrm{BH}}-\sigma$ relation and the evolution to the QSO phase.
\end{abstract}

Subject headings: galaxies: active — galaxies: formation — galaxies: nuclei — galaxies: starburst — infrared: galaxies

\footnotetext{
${ }^{1}$ National Astronomical Observatories, Chinese Academy of Sciences, Jia-20, DaTun Road, Chaoyang District, Beijing 100012, China

${ }^{2}$ Department of Astronomy, School of Physics, Peking Universty, Beijing 100871, China
} 


\section{Introduction}

Ultra-luminous infrared galaxies (ULIRGs) were discovered by Infrared Astronomical Satellite (IRAS) in large numbers with infrared luminosity in 8-1000 $\mu \mathrm{m}$ greater than $10^{12} L_{\odot}$. The serious intrinsic obscuration for ULIRGs in optical, UV, and even in midIR and X-ray bands (Condon et al. 1991b) makes it difficult to clearly probe their physical properties by observations. There are probably more ULIRGs at high redshifts than in the local universe, and even more than optically bright QSOs (Lonsdale et al. 2006). In the last two decades, multi-wavelength studies on ULIRGs have significantly improved our understanding of these dramatic objects (see the review papers of Sanders \& Mirabel 1996; Lonsdale et al. 2006).

Extremely high infrared luminosity of ULIRGs is dominated by starbursts, and sometimes with additional contribution from active galactic nuclei (AGNs). Most ULIRGs are interacting systems undergoing a wide range of merger stages (e.g., Zou et al. 1991; Clements et al. 1996; Murphy et al. 1996; Surace et al. 2000; Farrah et al. 2001; Veilleux et al. 2002). ULIRGs with large luminosity or spectroscopic signatures of AGNs are most likely late-stage mergers (Veilleux et al. 2002). Observations of the molecular gas in ULIRGs (see Mirabel \& Sanders 1988; Sanders et al. 1986, 1991; Gao \& Solomon 2004) proved that high-density gases are reserved in a compact nuclear region. The connection between ULIRGs and AGNs was also found from optical and mid-infrared spectra (e.g. Kim et al. 1998; Veilleux et al. 1997, 1999a, b). About $25 \%$ of ULIRGs present evidences of AGNs, and the percentage increases to $\sim 50 \%$ when $L_{\mathrm{IR}}$ is greater than $10^{12.3} L_{\odot}$. Less than $10 \%$ ULIRGs have broad emission lines, which are the so-called Type I ULIRGs (see Clements et al. 1996; Wu et al. 1998a, b;; Zheng et al. 1999; Canalizo \& Stockton 2001; Cui et al. 2001). ULIRGs and AGNs most probably have evolutionary connection. To understand such possible connection, Lonsdale et al. (2006) suggest that major mergers of gas-rich galaxies first form a massive cool starburst-dominated ULIRG, and then a warm ULIRG phase is followed when a central AGN turns on inside the dust cocoon and heats the surrounding dust. The central AGN will evolve into an optically bright phase when it blows away the surrounding dust cocoon. The resulting stellar system will resemble a spheroid, so that the mass of the central black hole $(\mathrm{BH})$ of the $\operatorname{AGN}\left(M_{\mathrm{BH}}\right)$ is related to the stellar velocity dispersion $\sigma$. The $M_{\mathrm{BH}}-\sigma$ relation might build up at that time. In this scenario, ULIRGs are in a pre-AGN phase. The typical BH mass of ULIRGs should be smaller, and the galaxy bulge may not have been constructed completely, compared with those of normal QSOs.

Kawakatu et al. (2006) used a sample of eight Type I ULIRGs in the local universe with data of full width at half-maximum (FWHM) of $\mathrm{H}_{\beta}$ and optical continuum luminosity $L_{\lambda}(5100 \AA)$ from Zheng et al. (2002) to estimate their BH masses and investigate the 
$\mathrm{BH}$-bulge relation. They found that the Type I ULIRGs have systematically smaller BH masses in spite of having the comparable bulge luminosity as QSOs and elliptical galaxies. We note that the FWHM of $\mathrm{H}_{\beta}$ given in Zheng et al. (2002) is the FWHM of the whole emission-line profile, not the FWHM of the broad-line component, which should be used in the estimation of the BH mass. Therefore, Kawakatu et al. (2006) may underestimate the masses of BHs for these ULIRGs. Using a sample of sources mostly from Zheng et al. (2002), Hao et al. (2005) carried out a study on the Type I ULIRGs (named as IR QSOs in their paper), and concluded that the typical BH mass of Type I ULIRGs is smaller, and the typical Eddington ratio $\left(L_{\mathrm{bol}} / L_{\mathrm{Edd}}\right)$ is larger than those of PG QSOs. At higher redshift, Borys et al. (2005) found that submillimeter galaxies have smaller BH masses than QSOs with respect to the same mass range of bulges. Alexander et al. (2008) concluded that submillimeter galaxies host BHs with a mass of $\log \left(M_{\mathrm{BH}} / M_{\odot}\right) \approx 7.8$ in their sample. Because local ULIRGs and high-redshift submillimeter galaxies are similar with each other, both having bright infrared luminosity and a large amount of gas, and locating in the interacting systems, the investigations on local ULIRGs could enlighten our understanding about the high-redshift submillimeter galaxies.

A large ULIRG sample is needed to study their central BHs, the BH-host galaxy relation, and galaxy evolution. The best-known samples of IRAS luminous infrared galaxies and ULIRGs are the Bright Galaxy Sample of Soifer et al. (1987), updated into the Revised Bright Galaxy Sample by Sanders et al. (2003), the complete flux-limited IRAS 1 Jy sample (Kim \& Sanders 1998), the 2 Jy sample of Strauss et al. (1990), and the FIRST/IRAS sample of Stanford et al. (2000). Since the Sloan Digital Sky Survey (SDSS) covers more than a quarter of the sky, more ULIRGs with relatively high quality spectra can be found by the cross-correlation of IRAS data with the SDSS. Goto (2005) investigated the optical properties of 4248 infrared galaxies with $L_{\mathrm{IR}}$ of $10^{9}$ to $10^{13.57} L_{\odot}$ from the cross-correlation between SDSS DR3 spectroscopic sample of galaxies and IRAS sources, and 181 of them are ULIRGs. Pasquali et al. (2005) used the SDSS DR2 data to study the optical properties of IRAS galaxies. Cao et al. (2006) identified 1207 luminous infrared galaxies and 57 ULIRGs from SDSS DR2 for a statistical study. Hwang et al. (2007) identified 324 ULIRGs from the SDSS, 2dF Galaxy Redshift Survey (Colless et al. 2001), and 6dF Galaxy Survey (Jones et al. 2004). Because the new SDSS spectroscopic sample of galaxies in DR6 has been released (Adelman-McCarthy et al. 2008), a larger ULIRG sample can be identified and then used to re-examine the statistical properties of ULIRGs.

This paper is organized as follows. In Section 2, we make a cross-identification between the IRAS Faint Source Catalog and the spectroscopic catalog of the SDSS DR6, and obtain a ULIRG sample. We separate them into two sub-samples, NL ULIRGs and Type I ULIRGs, and fit their SDSS spectra. We present the results and discuss the NL ULIRG sample in 
Section 3. In Section 4, we carry out a study on the BH masses and the $M_{\mathrm{BH}}-\sigma$ relation of Type I ULIRGs. The discussions and conclusions are presented in Sections 5 and 6. In this paper, we adopt $H_{0}=70 \mathrm{~km} \mathrm{~s}^{-1} \mathrm{Mpc}^{-1}, \Omega_{\mathrm{m}}=0.3$, and $\Omega_{\Lambda}=0.7$.

\section{The ULIRG sample}

SDSS DR6 spectra catalog contains about 750,000 galaxies and QSOs and covers over $7425 \mathrm{deg}^{2}$ (Adelman-McCarthy et al. 2008). IRAS Faint Sources Catalog (Moshir et al. 1992, hereafter FSC92) contains 173,044 sources with IR flux in bands of 12, 25, 60, and $100 \mu \mathrm{m}$. Although the IRAS data set was published more than 17 years ago, it is still very underexplored. Only $43 \%$ of the total IRAS extragalactic FSC sources have been included in any sort of publication (Lonsdale et al. 2006).

The positional uncertainty of IRAS source (about 1-13 arcsec for the in-scan direction and 3-55 arcsec for the cross-scan direction) is much larger than that of the object in the

SDSS DR6, and is described by an uncertainty ellipse. Similar to Hwang et al. (2007) and Cao et al. (2006), we used the positional uncertainty ellipse of each IRAS source to obtain their matched counterparts in the SDSS. If a galaxy of the SDSS DR6 falls into the $3 \sigma$ uncertainty ellipse of IRAS source, we regarded them as a match. As a result, we found that 11,354 IRAS sources have only one counterpart in the SDSS DR6 and 984 have more than one counterparts. In the later case, the likelihood ratio method (see Sutherland \& Saunders 1992; Hwang et al. 2007; Cao et al. 2006) is used to determine which counterpart of this $\operatorname{IRAS}$ source is the most probable one. After doing these, we obtained a list of $12,338 \operatorname{IRAS}$ sources with SDSS DR6 optical counterparts.

\subsection{Selection criteria}

Our selection criteria of ULIRGs are shown as follows: for the 12,338 matched IRAS sources, their $12 \mu \mathrm{m}$ and $25 \mu \mathrm{m}$ flux densities are mostly upper limits. Therefore, we calculated their far-infrared luminosity by using $60 \mu \mathrm{m}$ and $100 \mu \mathrm{m}$ fluxes with the following formulae (see Helou et al. 1988; Sanders \& Mirabel 1996) and converted it to the total infrared luminosity (Calzetti et al. 2000):

$$
\begin{gathered}
F_{\mathrm{FIR}}=1.26 \times 10^{-14}\left\{2.58 f_{60}+f_{100}\right\}\left(\mathrm{W} \mathrm{m}^{-2}\right), \\
L_{\mathrm{FIR}}=4 \pi D_{\mathrm{L}}^{2} F_{\mathrm{FIR}}\left(L_{\odot}\right) \\
L_{\mathrm{IR}}(1-1000 \mu m)=1.75 L_{\mathrm{FIR}} .
\end{gathered}
$$


Here $f_{60}, f_{100}$ are the $I R A S$ flux densities in Jy at 60 and $100 \mu m, D_{\mathrm{L}}$ is the luminosity distance, $F_{\mathrm{FIR}}$ is the far-infrared flux, $L_{\mathrm{FIR}}$ is the far-infrared luminosity, and $L_{\mathrm{IR}}$ is the infrared luminosity in $L_{\odot}$. For all the matched sources, we also required that the $60 \mu \mathrm{m}$ flux with high quality measurement (In the FSC92, high quality, moderate quality, and upper limits of the flux measurements are marked as 3, 2, and 1, respectively). Because the $100 \mu \mathrm{m}$ flux does not affect much on the value of $L_{\mathrm{IR}}$ (see Cao et al. 2006), we do not set the limit to the quality of the $100 \mu \mathrm{m}$ flux density. The redshift confidence of each source should be larger than 0.65. Finally we identified 325 ULIRG candidates with an IR luminosity greater than $10^{12} L_{\odot}$. When we check these 325 ULIRG candidates by using the NASA/IPAC Extragalactic Database (NED), we found 18 sources whose redshifts provided in the NED are not consistent with our results. According to the NED identifications, nine of these 18 sources have a Petrosian $r$-band magnitude less than 15, so they are too bright for the SDSS. If calculated their $L_{\mathrm{IR}}$ with the redshifts provided in the NED, we found that 17 of 18 sources are not ULIRGs. This discrepancy is probably due to the large position error of the IRAS sources and the incompleteness of the SDSS spectra. The fraction of the sources which are not excluded in the examination with the NED (307/325) is about $94.5 \%$, which is consistent with the reliability of our sample (about $93.4 \%$, see the next section). By excluding these 17 objects, we obtain a sample of 308 ULIRGs. Detailed information of our ULIRGs is given in Table 1.

The distributions of redshift and infrared luminosity of ULIRGs are shown in Figure 1. The redshifts of our ULIRG sample cover a range from about 0.03-0.6, with a median value of about 0.2 , which is similar to that of Hwang et al. (2007).

We compared our result with that of Hwang et al. (2007), who identified 126 ULIRGs from SDSS DR4 but adopted different methods to calculate $L_{\mathrm{IR}}$. Our catalog recovers 122 of their 126 ULIRGs. For the rest of the four sources in their sample, F07568+4823 and F11553+4557 have IR luminosities of $10^{11.99} L_{\odot}$ in our result and thus are not selected; F10200+4839 and F15239+4331 are not ULIRGs, and wrong optical counterparts were identified by Hwang et al. (2007).

In order to obtain the radio properties of these ULIRGs, we also cross identified our ULIRG sample with the NVSS (Condon et al. 1998) and FIRST (Becker et al. 1995; White et al. 1997) catalogs. Note that Best et al. (2005) used a hybrid NVSS-FIRST method to identify the radio counterparts for SDSS DR2 galaxies with high reliability and completeness. We followed their method to identify radio counterparts of our ULIRG sample, and found that 140 of 308 ULIRGs have counterparts in the NVSS catalog within a typical searching radius about $15^{\prime \prime}$, and 132 of these 140 sources have FIRST counterparts within $3^{\prime \prime}$. Some of them are probably core-dominant radio sources, such as F08201+2801, F13408+4047, F16413+3954, 
F10418+1153, F09105+4108, F13451+1232, F11206+3639 and F08507+3636. For the rest of the 168 ULIRGs, 82 of them have one FIRST counterpart within $3^{\prime \prime}$, and their radio flux densities are below or close to the NVSS flux limit (about $2.5 \mathrm{mJy}$ ). The radio information of these 222 ULIRGs is listed in our ULIRG catalog (see Table 1).

\subsection{Reliability estimated with the likelihood ratio method}

To estimate the reliability of our sample, we follow Cao et al. (2006) and Hwang et al. (2007) and adopt a likelihood ratio method (Sutherland \& Saunders 1992). The likelihood ratio $p$ is defined as

$$
p=\frac{Q\left(\leq m_{r}\right) \exp \left(-R^{2} / 2\right)}{2 \pi \sigma_{a} \sigma_{b} n\left(\leq m_{r}\right)},
$$

where $Q\left(\leq m_{r}\right)$ is the multiplicative factor measuring the probability for a true optical counterpart brighter than the flux limit exists in the association, and we set $Q=1$ for simplicity. $\sigma_{\mathrm{a}}$ and $\sigma_{\mathrm{b}}$ are the standard deviations, and $\mathrm{m}_{\mathrm{r}}$ is the SDSS $r$-band magnitude. Here we assume that the errors are Gaussian distributed(the error of an IRAS source is not a pure Gaussian, but in the statistical sense, the result of likelihood ratio study can still be used to evaluate the reliability), and define $R$ as

$$
R^{2}=\frac{\left(d_{1}\right)^{2}}{\sigma_{a 1}{ }^{2}+\sigma_{a 2}{ }^{2}}+\frac{\left(d_{2}\right)^{2}}{\sigma_{b 1}{ }^{2}+\sigma_{b 2}{ }^{2}},
$$

where $d_{1}$ and $d_{2}$ are the positional differences along the two axes of the error ellipse between each IRAS source and its SDSS counterpart, $\sigma_{\mathrm{a} 1}$ and $\sigma_{\mathrm{b} 1}$ are the errors of each IRAS source along the $x$ - and $y$-axes, $\sigma_{\mathrm{a} 2}$ and $\sigma_{\mathrm{b} 2}$ are the errors of each matched SDSS source along the $x$ - and $y$-axes. Because the positional error of an SDSS source is much smaller than that of an IRAS source, we only consider $\sigma_{\mathrm{a} 1}$ and $\sigma_{\mathrm{b} 1}$.

In this work, we adopt the $3 \sigma$ error ellipse as the match justification; thus $n\left(\leq m_{r}\right)$ can be obtained by using the formula:

$$
n\left(\leq m_{r}\right)=\frac{N\left(\leq m_{r}\right)}{9 \pi \sigma_{a 1} \sigma_{b 1}},
$$

where $n\left(\leq m_{r}\right)$ is the total surface density of objects brighter than the candidate, and $N\left(\leq m_{r}\right)$ is the number of galaxies whose magnitude is less than or equal to $\mathrm{m}_{\mathrm{r}}$. Under the above considerations, we obtain

$$
p=\frac{9 \exp \left(-R^{2} / 2\right)}{2 N\left(\leq m_{r}\right)},
$$


where we use the $r$-band Petrosian magnitude for galaxies of the SDSS DR6 to calculate $p$ of each source. To obtain the reliability, we adopt the method proposed by Lonsdale et al. (1998) and Masci et al. (2001). The reliability of a source with $p$ is given by

$$
R e(p)=1-\frac{N_{\text {random }}(p)}{N_{\text {true }}(p)},
$$

where $N_{\text {true }}(p)$ represents the number of true associations, and $N_{\text {random }}(p)$ represents the number of random associations with a $p$, which can be derived by offsetting the positions of IRAS sources and re-calculating the associated sample. The numbers of true and randommatched sources are 12,338 and 813, and then the reliability of our sample is about $93.4 \%$. Thus we believe that our ULIRG sample is reliable enough to make a statistical study on the properties of NL ULIRGs and Type I ULIRGs. The distribution of the reliabilities for our 308 ULIRGs is shown in Figure 2 ,

\subsection{The optical images of ULIRGs}

We examine the images of these 308 ULIRGs by using the SDSS DR6 Image List Tool. Due to the limited resolution of the SDSS image, we can only mark the ULIRGs with an obvious interaction feature. ULIRGs are classified into (see Veilleux et al. 2002) class I with one nucleus but with tail features; class II have two identified nuclei and well-developed tidal tails or/and bridges; class III have two close or even overlapped nuclei, and their redshifts (almost in all cases, only one source in this system has spectral redshift measurements, and another/others have only SDSS photometric redshift data) are consistent. Examples are shown in Figure 3, About 56\% of the ULIRGs in our total sample show obvious interaction features. The nearby ULIRGs show more clearly the interaction features, with the percentage of about $92 \%$ for $z<0.1,84 \%$ for $z<0.15$, and $79 \%$ for $z<0.2$. The selection effect is obvious in the classification of interaction features of the ULIRGs, because we can see the tails and two interacting galaxies at the maximum redshift about $z=0.3$. Objects at various merger stages appear in our ULIRG sample. Some of them are still widely separated, and some are advanced mergers. The minimum separation between two nuclei in our sample is about $2^{\prime \prime}$. The imaging property is classified for each source in Table 1.

Optical spectra of all 308 ULIRGs are available in the SDSS archive. This is the largest sample of ULIRGs with optical spectra. 

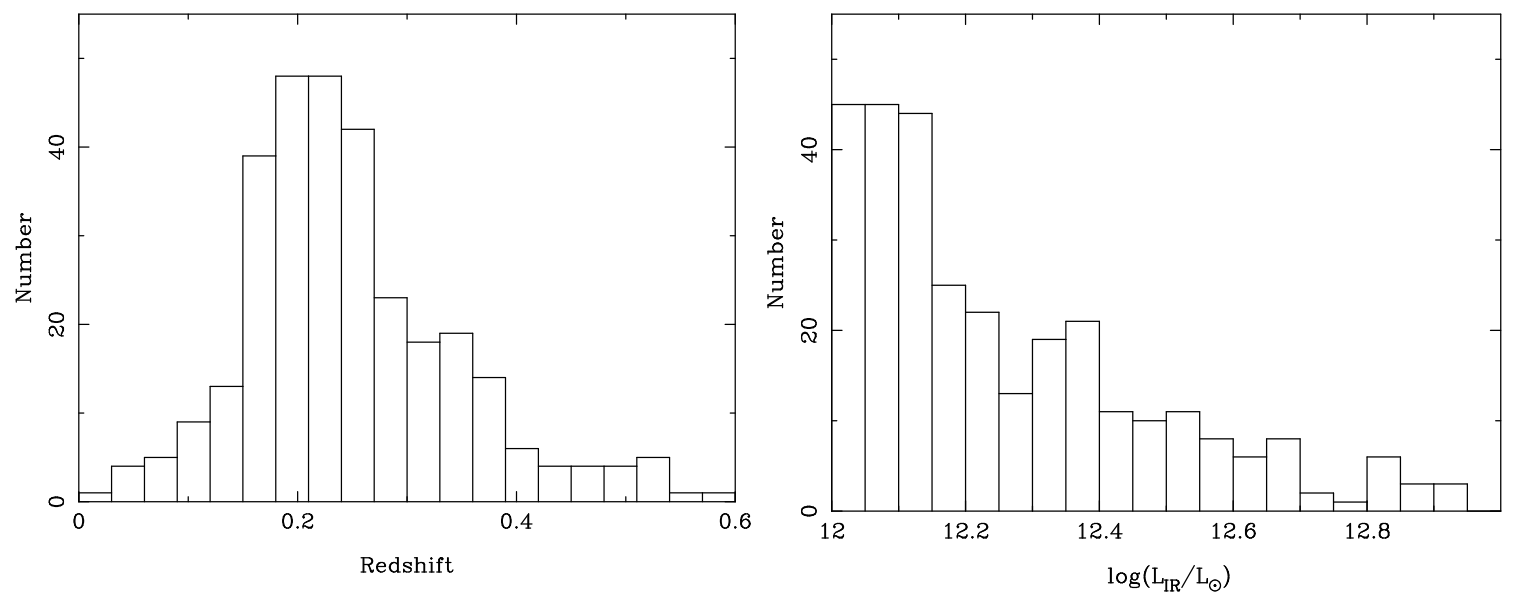

Fig. 1.- Distribution of redshift (left panel) and $L_{\mathrm{IR}}$ (right panel) of our ULIRG sample.

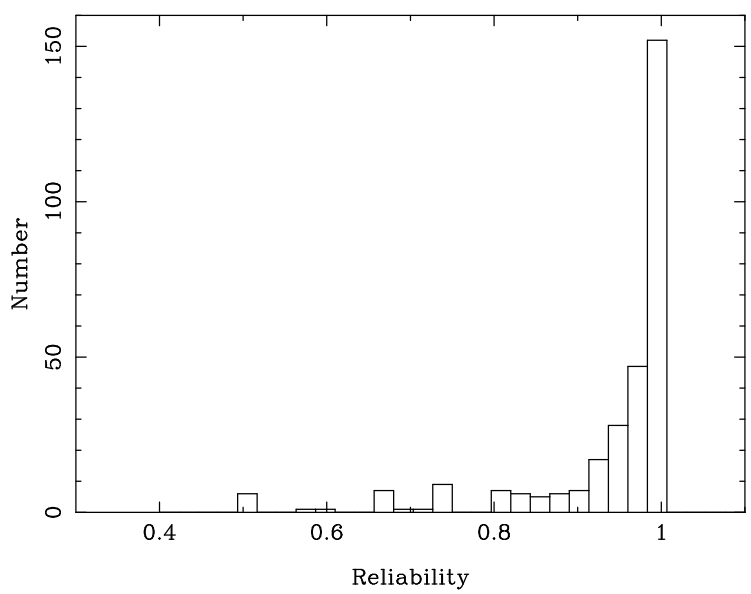

Fig. 2.- Distribution of source matching reliabilities of our ULIRG sample. 


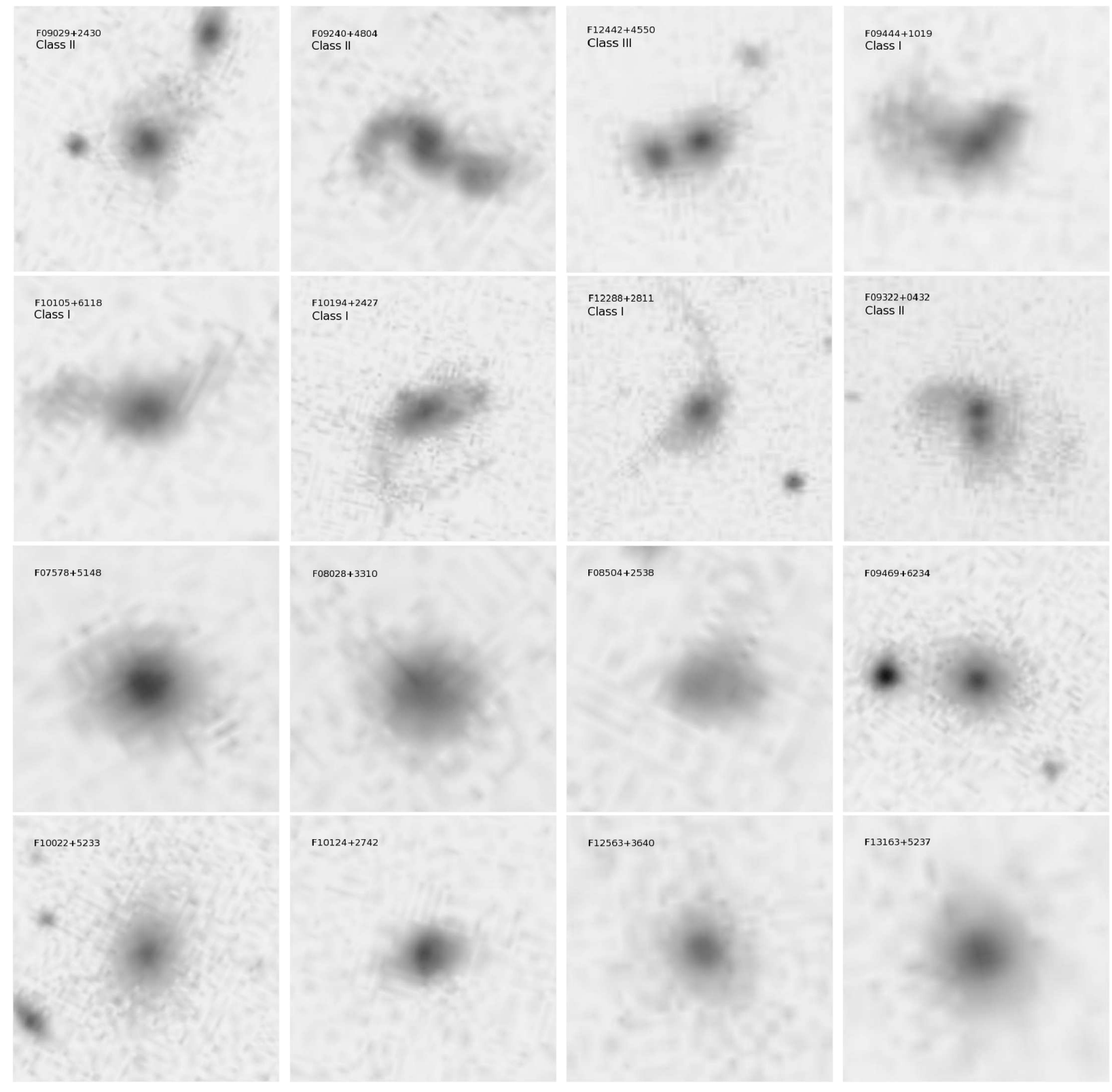

Fig. 3.- Examples of SDSS images of ULIRGs with (the upper eight ) or without (the lower eight) obvious interacting features in our sample. The interaction classifications are labeled for the upper eight ULIRGs. 


\subsection{Optical spectra of ULIRGs}

We want to identify Type I ULIRGs and NL ULIRGs from this sample. Only the spectra with signal-to-noise $(\mathrm{S} / \mathrm{N})>3$ are considered in this study. After excluding the sources with no obvious emission lines in their spectra, we found 209 sources with emission lines, 62 of which have broad-line components and are Type I ULIRGs.

SDSS spectra were processed as follows: first, we correct the Galactic extinction and redshift effects, and then subtract the $\mathrm{Fe}_{\text {II }}$ emissions from the continuum by use of the optical iron template from Boroson \& Green (1992) in the wavelength range $4250 \AA<\lambda<$ $7000 \AA$. Second, we fit the spectra using the Mpfit package in IDL which is based on the Levenberg-Marquardt method. The continuum emission of ULIRGs comes from central AGN and host galaxy, and is often modified by the intrinsic dust extinctions. These effects should be considered for the determination of the continuum flux. A local power law is used

for fitting the continuum. After the subtraction of the fitted continuum emission from the spectra, we fit the emission lines. For NL ULIRGs, we use a single Gaussian profile to fit

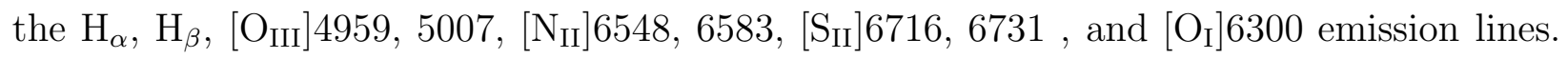
When a single Gaussian cannot fit the profile of emission lines very well, double Gaussians are used to obtain the line flux. For Type I UILRGs, we use two Gaussian components to fit $\mathrm{H}_{\alpha}$ and $\mathrm{H}_{\beta}$. If the [O $\mathrm{O}_{\mathrm{III}}$ ] emission lines can not be well fitted by a single Gaussian, two Gaussian components are also used. Examples of the fitted spectra are given in Figure 4 .

\section{NL ULIRGs}

The emission-line properties of 147 NL ULIRGs in our sample can be obtained and are listed in Table 1. We first classify them using the Baldwin-Phillips-Terlevich (BPT) diagram (Baldwin et al. 1981, see Figure 5).

The Balmer decrement method is often used to evaluate the intrinsic reddening effect. In previous works (e.g. Kewley et al. 2006; Veilleux et al. 1999a, b), $\mathrm{H}_{\alpha} / \mathrm{H}_{\beta}=2.85$ was used for galaxies whose emissions are dominated by star formation, and $\mathrm{H}_{\alpha} / \mathrm{H}_{\beta}=3.1$ is used for galaxies dominated by the AGN. But before we classify the object through the BPT diagrams, we can not assess which source is dominated by the star formation or the AGN. In this work, we adopt the intrinsic ratio $\mathrm{H}_{\alpha} / \mathrm{H}_{\beta}=3.1$, and the reddening curve of Cardelli et al. (1989, hereafter CCM89), and also assume $R_{v}=A_{v} / E(B-V)=3.1$ to do the optical classification. In order to examine the influence of the intrinsic ratio of $\mathrm{H}_{\alpha} / \mathrm{H}_{\beta}$, we also used $\mathrm{H}_{\alpha} / \mathrm{H}_{\beta}=2.85$ to do the classification again, and found that the classification of only one source (F09444+1019) is not consistent with the case of assuming $\mathrm{H}_{\alpha} / \mathrm{H}_{\beta}=3.1$. 

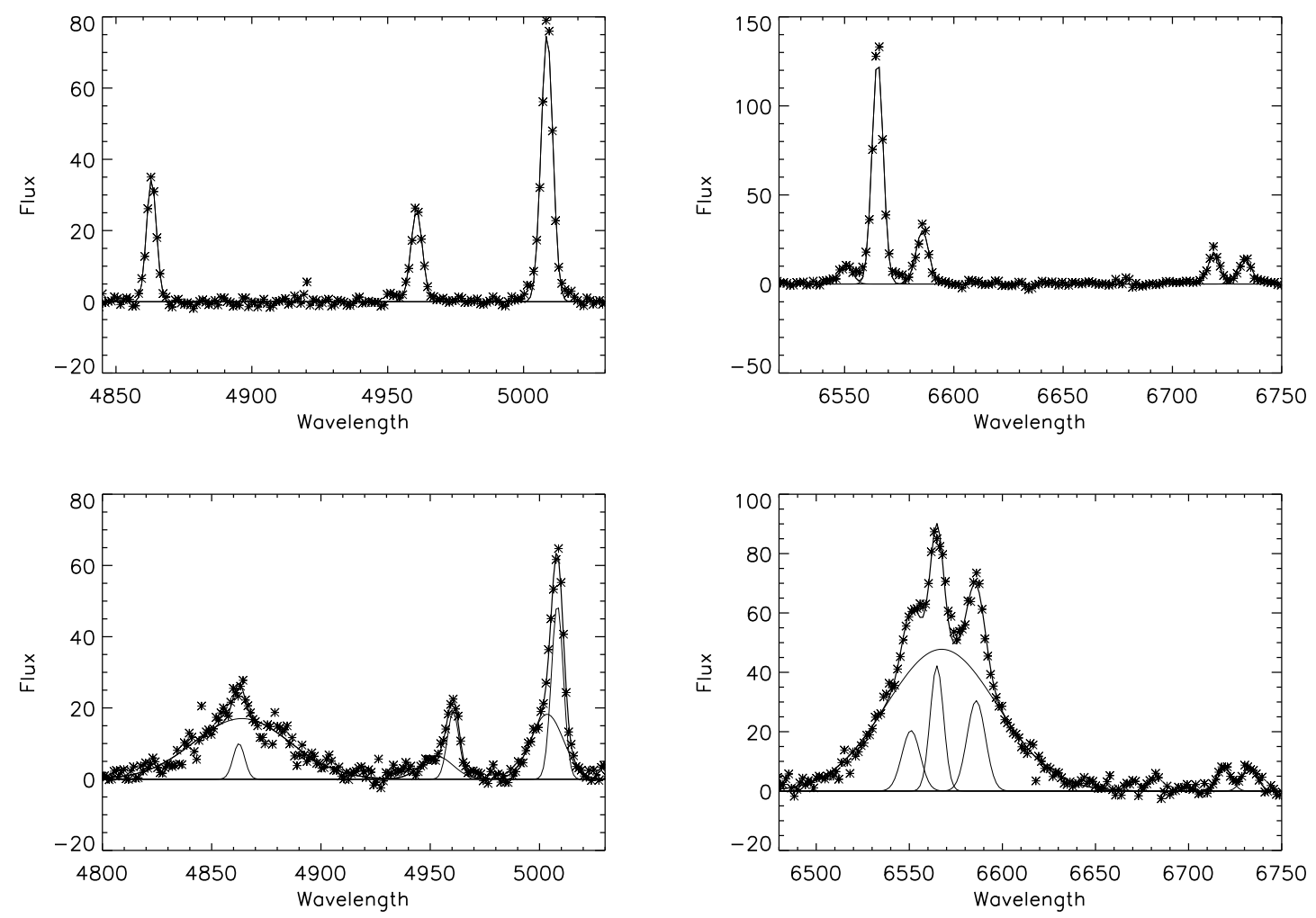

Fig. 4. - Examples of our fitted SDSS spectra for the $\mathrm{H}_{\beta}$ and $\mathrm{H}_{\alpha}$ regions for a NL ULIRG (two upper panels) and a Type I ULIRG (two lower panels) 
The intrinsic flux at wavelength $\lambda$ can be expressed as

$$
I(\lambda)=F(\lambda) \times 10^{c f(\lambda)} .
$$

The relation between the intrinsic and observed line ratio of $\mathrm{H}_{\alpha}$ and $\mathrm{H}_{\beta}$ is

$$
I\left(H_{\alpha}\right) / I\left(H_{\beta}\right)=10^{c\left(f\left(H_{\alpha}\right)-f\left(H_{\beta}\right)\right)} \times F\left(H_{\alpha}\right) / F\left(H_{\beta}\right),
$$

where $f(\lambda)$ is related to the generalized CCM89 reddening curve, $I(\lambda)$ is the intrinsic flux, $F(\lambda)$ is the observed flux, and $c$ is the value of the Balmer extinction. We estimated $c$ from the observed line ratio and the reddening curve, after the intrinsic line ratio is assumed. We use the new classification scheme made by Kewley et al. (2006) for the classification.

The 147 NL ULIRGs are classified as five types: 29 star-forming galaxies, 62 composite galaxies (which are likely to contain metal-rich stellar populations, plus AGN, see Kewley et al. 2006), 34 Seyfert galaxies, 6 LINERs, and 16 ambiguous galaxies, which are classified as one type in one or two diagrams but another type in the other diagram(s). Together with the 62 Type I ULIRGs, the percentage for AGNs is about $78 \%$ if we regard that the composite galaxies also contain AGNs. The AGN percentage becomes $49 \%$ if we only consider the Seyfert galaxies, LINERs, and Type I ULIRGs. The AGN percentage of ULIRGs increases with the infrared luminosity, consistent with the results in previous works (e.g. Veilleux et al. 1995, 1997; Wu et al. 1998b; Cao et al. 2006). The percentage (only consider the Seyfert galaxies, LINERs, and Type I ULIRGs as AGNs) is $71 \%$ for ULIRGs with $L_{\mathrm{IR}}>12.3 L_{\odot}$, and $89 \%$ for $L_{\mathrm{IR}}>12.4 L_{\odot}$.

A tight correlation between far-infrared and radio luminosities, covering about four orders of magnitude in the $L_{\mathrm{IR}}$, has been found for infrared-selected galaxies (e.g. Helou et al. 1985; Yun et al. 2001). The non-thermal radiation from the relativistic electrons related to the supernova remnants is believed to be the main reason of this relation. Here, we verify this relation using our ULIRG sample. The FIRST flux density was used to calculate the radio luminosity. In our sample, 214 ULIRGs have FIRST counterparts, including 118 NL ULIRGs and 45 Type I ULIRGs.

The luminosities at $60 \mu \mathrm{m}$ and at $1.4 \mathrm{GHz}$ radio band are obtained using the following formulae (Yun et al. 2001):

$$
\begin{gathered}
\log L_{1.4 \mathrm{GHz}}\left(\mathrm{W} \mathrm{Hz}^{-1}\right)=20.08+2 \log \mathrm{D}+\log \mathrm{S}_{1.4 \mathrm{GHz}}, \\
\log L_{60 \mu \mathrm{m}}\left(L_{\odot}\right)=6.014+2 \log D+\log S_{60 \mu m},
\end{gathered}
$$

where $D$ is the luminosity distance in $\mathrm{Mpc}, S_{1.4 \mathrm{GHz}}$ and $S_{60 \mu \mathrm{m}}$ are flux densities in units of Jy. The radio-FIR relation of our ULIRGs is shown in Figure 6. To examine the deviation of 

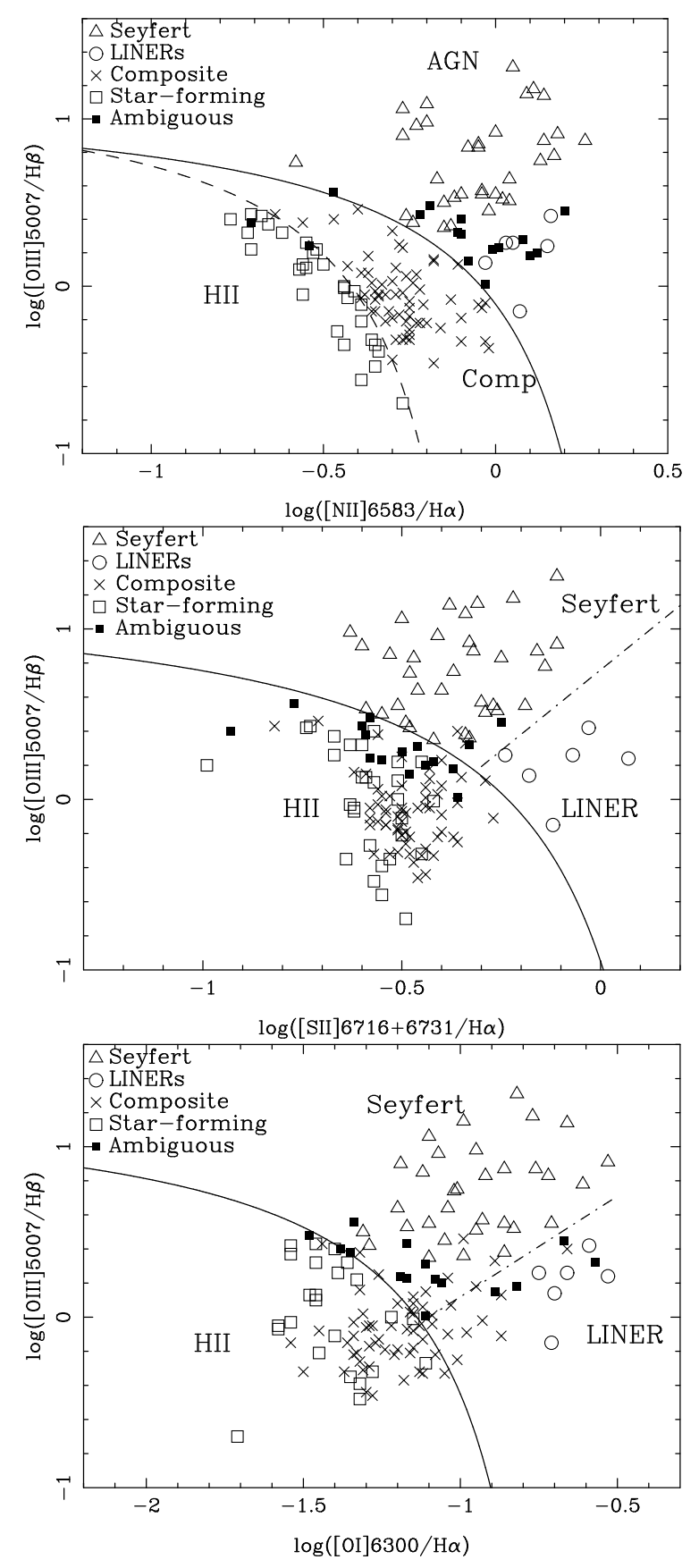

Fig. 5.- BPT diagrams for 147 NL ULIRGs. The Balmer decrement method has been used to derive the extinction corrected flux of each emission line. Upper panel: the $\left[\mathrm{N}_{\mathrm{II}}\right] / \mathrm{H}_{\alpha}$ vs. $\left[\mathrm{O}_{\mathrm{III}}\right] / \mathrm{H}_{\beta}$ diagnostic diagram. The solid line is from Kewley et al. (2006), the dashed line is from Kauffmann et al. (2003). Middle panel: the $\left[\mathrm{S}_{\mathrm{II}}\right] / \mathrm{H}_{\alpha}$ vs. $\left[\mathrm{O}_{\mathrm{III}}\right] / \mathrm{H}_{\beta}$ diagnostic diagram. The two lines are from Kewley et al. (2006). Lower panel: The $\left[\mathrm{O}_{\mathrm{I}}\right] / \mathrm{H}_{\alpha}$ vs. $\left[\mathrm{O}_{\mathrm{III}}\right] / \mathrm{H}_{\beta}$ diagnostic diagram. The two lines are from Kewley et al. (2006). 
these ULIRGs from the linear relation, we also calculated the $q$ parameter (see Condon et al. 1991a; Yun et al. 2001),

$$
q=\log \left(\frac{2.58 S_{60 \mu \mathrm{m}}+S_{100 \mu m}}{2.98 \mathrm{Jy}}\right)-\log \left(\frac{S_{1.4 \mathrm{GHz}}}{\mathrm{Jy}}\right) .
$$

A plot of $q$ versus $L_{60 \mu \mathrm{m}}$ is shown in Figure[7. The radio excess objects (i.e., the objects having 3 times larger radio flux density than the expected values from the linear radio-FIR relation of Yun et al. (2001)) are AGNs, either Type I ULIRGs, or Seyfert galaxies. Therefore, the radio-FIR relation originates from the starburst-related non-thermal radiation, and the radio excess objects are due to the AGN-related radio emission (Roy \& Norris 1997).

\section{Type I ULIRGs}

In our ULIRG sample, there are 62 Type I ULIRGs. We can explore their properties of central BHs. Examples of their SDSS spectra are shown in Figure 8 . The parameters of these Type I ULIRGs are listed in Table 2.

\subsection{Black hole masses of Type I ULIRGs}

We assume that the motion of the gas moving around the central $\mathrm{BH}$ is dominated by the gravitation force and that the gas of broad emission line region (hereafter BLR) is virialized (e.g. Peterson \& Wandel 1999, 2000). The BH mass can then be expressed as $M_{\mathrm{BH}}=\frac{R_{B L R} V^{2}}{G}$, and the realistic formula given by Kaspi et al. (2000) is:

$$
\frac{M_{\mathrm{BH}}}{M_{\odot}}=1.464 \times 10^{5}\left(\frac{R_{\mathrm{BLR}}}{\mathrm{lt}-\text { days }}\right)\left(\frac{v_{\mathrm{FWHM}}}{10^{3} \mathrm{~km} \mathrm{~s}^{-1}}\right)^{2},
$$

where $v_{\mathrm{FWHM}}$ is the FWHM of the broad emission line, and $R_{\mathrm{BLR}}$ is the radius of the BLR. For our objects, the $v_{\mathrm{FWHM}}$ is taken as the FWHM of the broad component of the $\mathrm{H}_{\beta}$ emission line, and the $R_{\mathrm{BLR}}$ can be estimated from the $\mathrm{Fe}_{\mathrm{II}}$ and the Galactic-extinction-corrected continuum luminosity at $5100 \AA$, using:

$$
\frac{R_{\mathrm{BLR}}}{\mathrm{lt}-\text { days }}=(26.4 \pm 4.4)\left[\frac{\lambda L_{\lambda}(5100 \AA)}{10^{44} \mathrm{erg} \mathrm{s}^{-1}}\right]^{(0.61 \pm 0.10)} \text {. }
$$

The relation between $R_{\mathrm{BLR}}$ and $L_{\lambda}(5100 \AA)$ was first found by Kaspi et al. (2000), and their data were refitted by McLure \& Jarvis (2002) in the same cosmology as we adopted. Here we assume that the central AGN dominates the continuum emission at $5100 \AA$, and the contribution from stellar emission and the intrinsic reddening effect can be neglected. Kawakatu et al. 


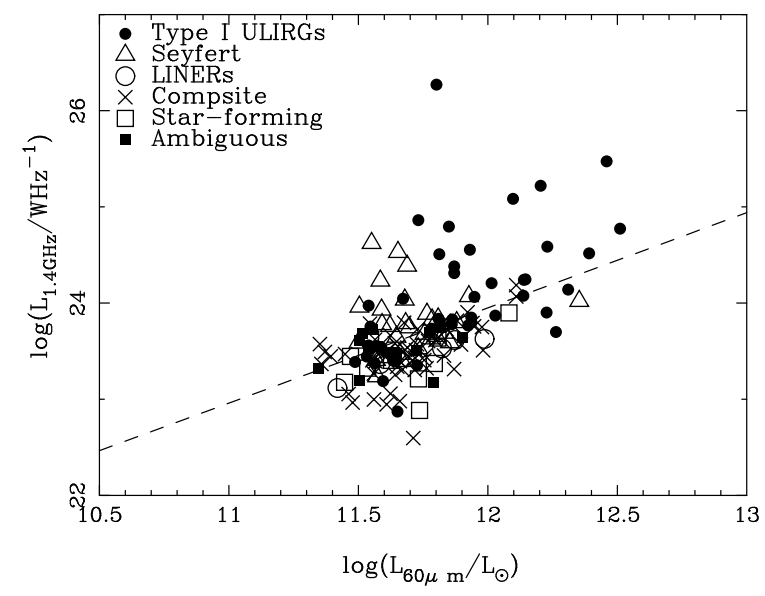

Fig. 6. - $1.4 \mathrm{GHz}$ radio luminosity vs. IRAS $60 \mu \mathrm{m}$ luminosity for our NL ULIRGs and Type I ULIRGs. The dashed line is the best fit given by Yun et al. (2001) from 1809 infrared galaxies.

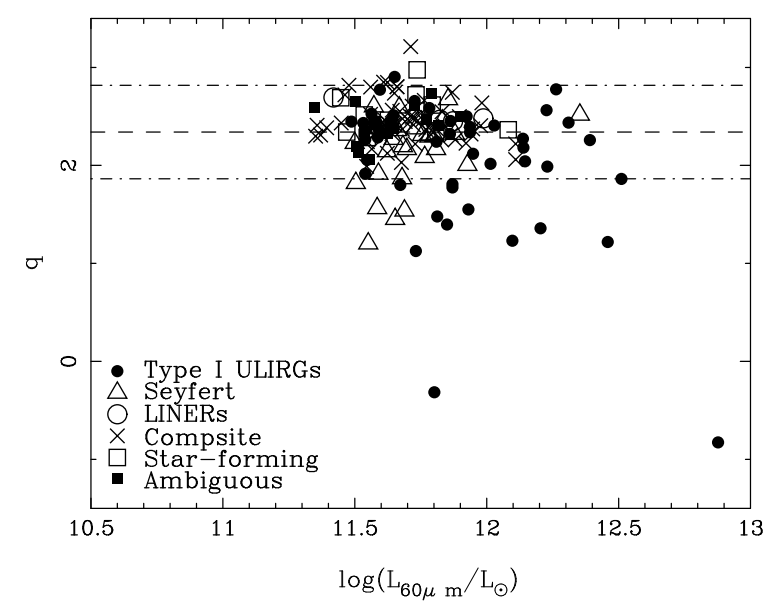

Fig. 7.- The $q$-values plotted against the IRAS $60 \mu \mathrm{m}$ luminosities for the same sample used in Figure 6. The dashed line marks the average value of $q=2.34$ obtained by Yun et al. (2001) from a sample of 1809 infrared galaxies. The two dot-dashed lines represent a three times larger radio (lower line) or IR (upper line) flux density than the expected values from the linear radio-FIR relation of Yun et al. (2001). 

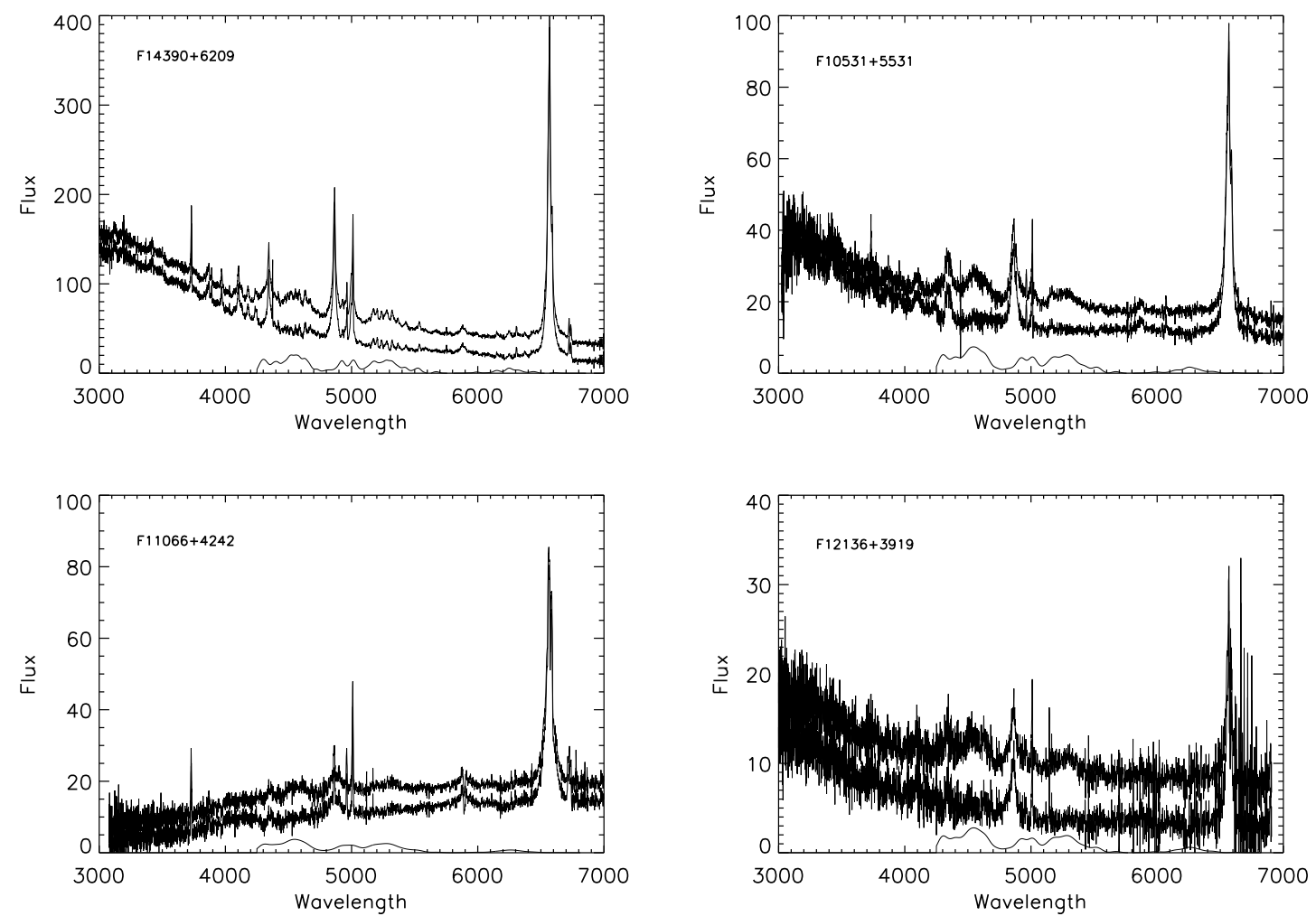

Fig. 8.- Examples of SDSS spectra of Type I ULIRGs in our sample. In each panel, the top curve is the extinction and redshift-corrected spectra. The middle curve is the spectra after the subtraction of the $\mathrm{Fe}_{\mathrm{II}}$ emissions, which was shifted downward. The bottom curve is the model for $\mathrm{Fe}_{\mathrm{II}}$ emissions. 
(2006) argued that, for Type I ULIRGs, the central AGN dominance of the $5100 \AA$ emission is reasonable.

To investigate the BH masses of Type I ULIRGs systematically, a large sample is absolutely needed. Kawakatu et al. (2006) used eight ULIRGs from the Type I ULIRG sample of Zheng et al. (2002) to investigate this problem, and concluded that the BH masses of Type I ULIRGs are typically smaller than those of PG QSOs. The FWHM $\mathrm{H}_{\beta}$ they used is not the broad component of $\mathrm{H}_{\beta}$, which leads to an underestimation of the $\mathrm{BH}$ mass. Hao et al. (2005) carried out a study on Type I ULIRGs, with a sample mainly from Zheng et al. (2002), but added several PG QSOs. The FWHM of the $\mathrm{H}_{\beta}$ broad component they used was estimated in the same way as that in Boroson \& Green (1992), who used double components to fit the $\mathrm{H}_{\beta}$ line. Hao et al. (2005) used the cosmology of $H_{0}=70 \mathrm{~km} \mathrm{~s}^{-1} \mathrm{Mpc}^{-1}, \Omega_{\mathrm{m}}=0.3$, and $\Omega_{\Lambda}=0.7$, the same as ours. We noted that three of our 62 Type I ULIRGs were listed in their sample: for F01572+0009, the BH mass in our work $\left(\log \left(M_{\mathrm{BH}} / M_{\odot}\right) \sim 8.0\right)$ is consistent with their result $\left(\log \left(M_{\mathrm{BH}} / M_{\odot}\right) \sim 7.7\right)$. For F10026+4347, the same $\mathrm{BH}$ mass $\left(\log \left(M_{\mathrm{BH}} / M_{\odot}\right) \sim 7.8\right)$ is given in Hao et al. (2005) and our work. But for the other source F13342+3932, the BH mass we obtained $\left(\log \left(M_{\mathrm{BH}} / M_{\odot}\right) \sim 8.4\right)$ is larger than theirs $\left(\log \left(M_{\mathrm{BH}} / M_{\odot}\right) \sim 7.4\right)$, mainly because we use the much more broad component of $\mathrm{H}_{\beta}$ from SDSS spectra.

We combine the sample of Hao et al. (2005) with ours, and obtain a large sample of 90 Type I ULIRGs. The distribution of the BH mass of this sample is shown in Figure 9 , together with that of PG QSOs. Obviously, the BH masses of Type I ULIRGs (with a mean value of $6.7 \times 10^{7} M_{\odot}$ for these 90 Type I ULIRGs and about $7.6 \times 10^{7} M_{\odot}$ for our 62 Type I ULIRGs) are systematically smaller than those of PG QSOs (with a mean value of $2 \times 10^{8} M_{\odot}$ ), consistent with the popular evolutionary scheme that ULIRGs are in a pre-QSO phase and their central BHs are still under growing.

The bolometric luminosity $L_{\text {bol }}$ measures the total luminosity associated with the AGN. We estimate the bolometric luminosity using the formula in Kaspi et al. (2000): $L_{\mathrm{bol}} \approx 9 \lambda L_{\lambda}(5100 \AA)$. For those ULIRGs from Hao et al. (2005), we directly use their data because they used the same method to calculate the $\mathrm{BH}$ mass and $L_{\mathrm{bol}}$. The distribution of the Eddington ratio of this sample is shown in Figure 10, and the mean value is about 0.92 for these 90 Type I ULIRGs, and 0.55 for our 62 Type I ULIRGs, larger than that of PG QSOs (the mean value of the Eddington ratio for them is about 0.2).

The intrinsic reddening effect in Type I ULIRGs is still poorly known, and it probably affects the estimation of the optical luminosity. The Balmer decrement method is often used to estimate the reddening effect for narrow emission galaxies, but is seldom used for broad emission-line galaxies. In principle, the observations in the X-ray band can be used to estimate the absorption column density and then the absorption. But for our 62 Type I 


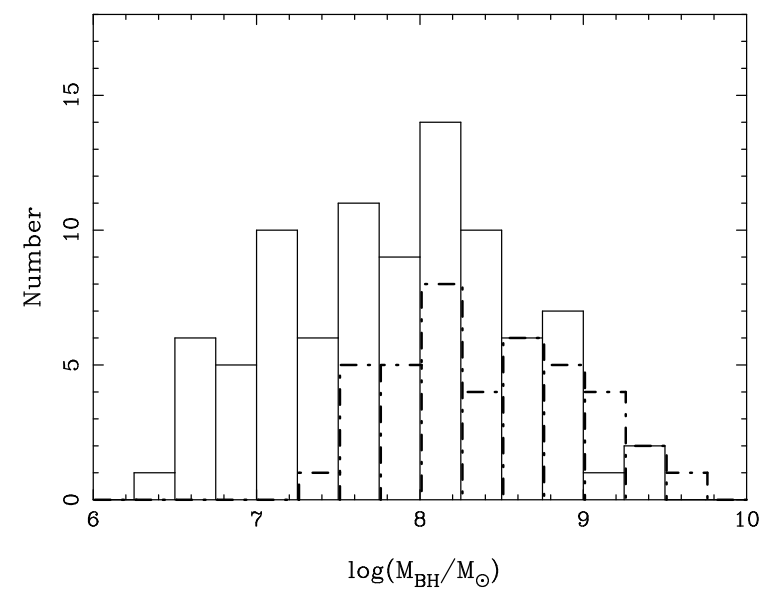

Fig. 9.- BH mass distribution. The solid line represents the $\mathrm{BH}$ mass distribution of our sample and the Type I ULIRGs from Hao et al.(2005), while the dot-dashed line is for that of the PG QSOs obtained from Hao et al.(2005).

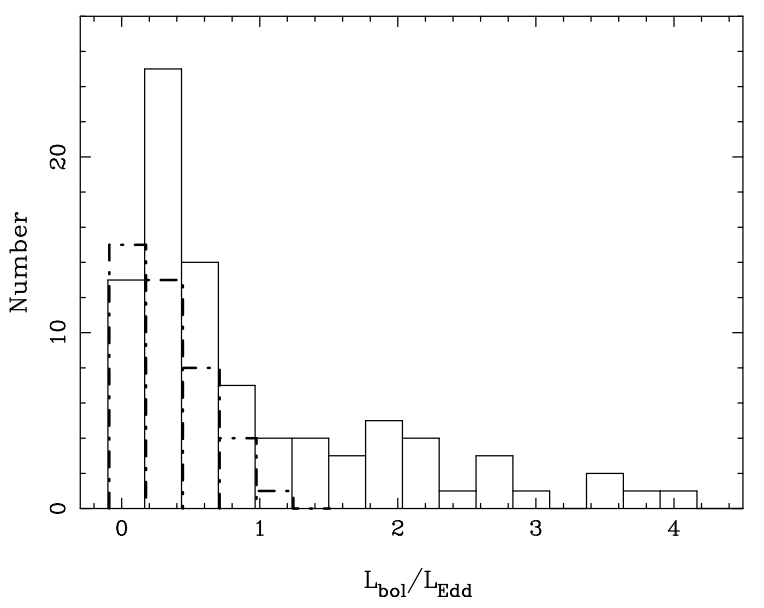

Fig. 10. - Eddington ratio distribution of 90 Type I ULIRGs from our sample and Hao et al. (2005), while the dot-dashed line is for that of the PG QSOs obtained from Hao et al.(2005). 
ULIRGs, only several have been observed in the X-ray band. More work is still needed to understand the intrinsic reddening effect in Type I ULIRGs.

\subsection{The $M_{\mathrm{BH}}-\sigma$ relation for Type I ULIRGs}

The $M_{\mathrm{BH}}-\sigma$ relation for ULIRGs was seldom discussed in the past, mainly due to the poor understanding of BH masses and the limitation of the sample size. Dasyra et al. (2006b) measured the velocity dispersions of 54 ULIRGs, and carried out a simulation to study the reasonableness of using the $M_{\mathrm{BH}}-\sigma$ relation to estimate their $\mathrm{BH}$ masses. They concluded that if the efficiency of gas accretion onto the $\mathrm{BH}$ from its surroundings remains constant with time, this relation can be used. For Type I ULIRGs, we can estimate their BH masses and velocity dispersions by use of their optical spectra, thus it is possible to test the $M_{\mathrm{BH}}-\sigma$ relation for ULIRGs by observation. Here we investigate the $M_{\mathrm{BH}}-\sigma$ relation for our Type I ULIRG sample.

When the stellar velocity dispersions have measured data in the literature, we adopt them directly, otherwise, the line width of some narrow emission lines, such as $\left[\mathrm{O}_{\mathrm{III}}\right] 5007$, $\left[\mathrm{O}_{\mathrm{III}}\right] 5007 \mathrm{NL}$ core, [ $\left.\mathrm{S}_{\mathrm{II}}\right] 6716$ or 6731 , are used to be the surrogates for $\sigma$, which were usually adopted in the studies on different types of AGNs (e.g. Nelson 2000; Boroson 2003; Grupe \& Mathur 2004; Salviander et al. 2007; Komossa \& Xu 2007). For six sources, F01572+0009, F12540+5708, F15462-0450, F21219-1757, F13451+1232(w), and PG 0050+124, their velocity dispersions have been measured in Dasyra et al. (2006a, b, 2007), and are directly adopted here. $\mathrm{BH}$ mass uncertainty is estimated from the uncertainty of the formula Equation (15) and the errors of the $L_{\lambda}(5100 \AA)$ and $\mathrm{FWHM}_{\mathrm{H}_{\beta}}$. The uncertainty of $\sigma$ is estimated from the fitting error of FWHM of emission lines, and the instrumental resolution for SDSS spectra is about $70 \mathrm{~km} \mathrm{~s}^{-1}$. To make a comparison, we also use the value of seven QSOs from Dasyra et al. (2007), who measured the $\sigma$ for 11 QSOs. Among these 11 QSOs, PG0050+124 is identified as a Type I ULIRG and PG1404+226 as a NL Seyfert 1 galaxy in Hao et al. (2005). PG1426+015 is an interacting system. The BH mass of LBQS0307-0101 is unavailable. Thus only seven of 11 QSOs are used. The instrumental resolution for their measured velocity dispersion is about $30 \mathrm{~km} \mathrm{~s}^{-1}$.

We show the $M_{\mathrm{BH}}-\sigma$ relation of our sample by using these surrogates of $\sigma$ in Figure 11, where $\sigma=\mathrm{FWHM}_{\text {lines }} / 2.35$. Because the outflow may influence the profile of $\left[\mathrm{O}_{\mathrm{III}}\right]$, there are some problems when using the FWHM of the $\left[\mathrm{O}_{\mathrm{III}}\right] 5007$ profile as the surrogate of $\sigma$. Thus we do not adopt the FWHM of the $\left[\mathrm{O}_{\mathrm{III}}\right] 5007$ profile as the surrogate of $\sigma$. In the upper panel, the FWHM of the [O $\left.\mathrm{O}_{\mathrm{III}}\right]$ NL core is used to estimate $\sigma$. Komossa \& Xu (2007) used $\left[\mathrm{S}_{\mathrm{II}}\right]$ as the surrogate of $\sigma$ and found that NL Seyfert $1 \mathrm{~s}$ do follow the $M_{\mathrm{BH}}-\sigma$ relation. In the 

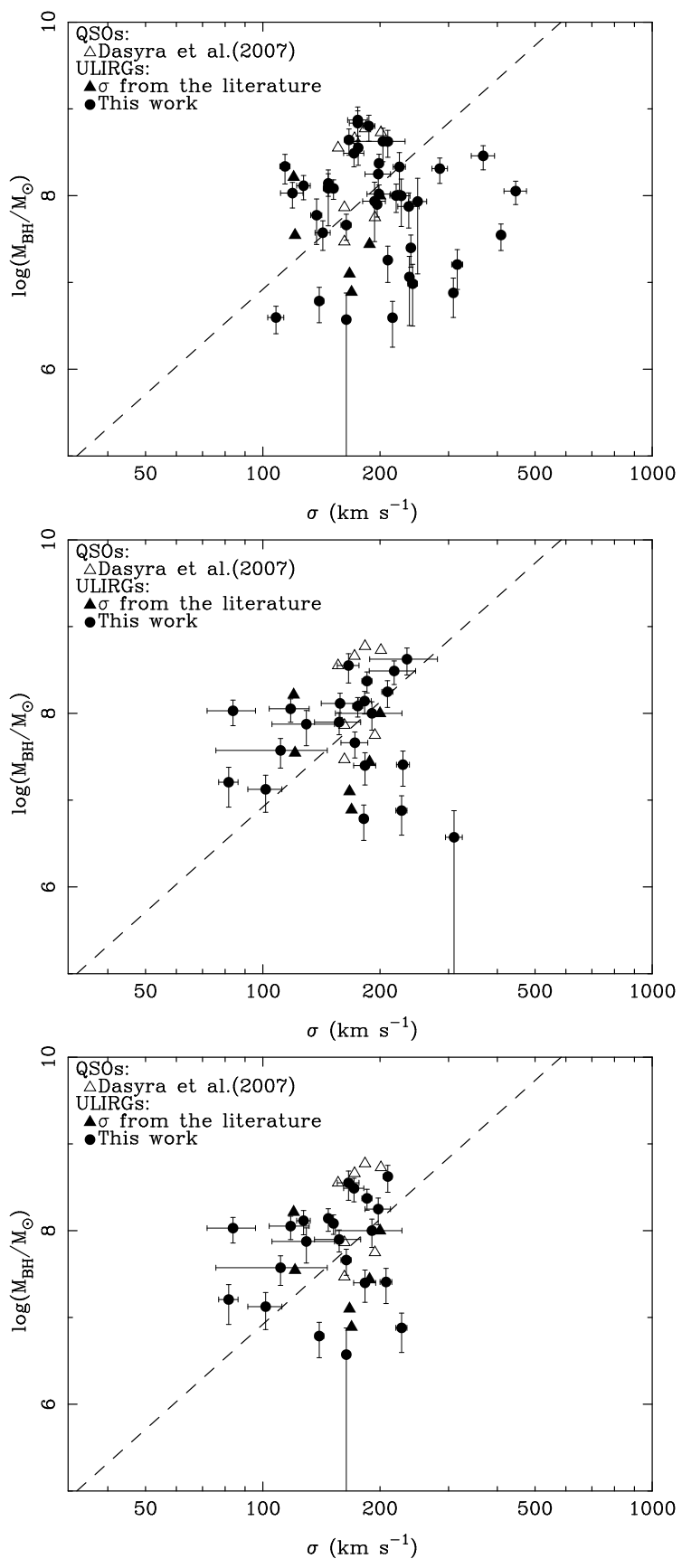

Fig. 11. $-M_{\mathrm{BH}}-\sigma$ diagrams for Type I ULIRGs. The filled triangles represent the six sources with measured $\sigma$ in Dasvra et al. (2006a, b, 2007), open triangles represent the seven QSOs with measured $\sigma$ in Dasyra et al. (2007), filled circles indicate the ULIRGs whose parameters are estimated in this work. If $\sigma$ is unavailable in reference, it was estimated by several methods: in the upper panel, the $\sigma$ is estimated from the FWHM of the $\left[\mathrm{O}_{\mathrm{III}}\right] \mathrm{NL}$ core; in the middle panel, the $\sigma$ is estimated from the FWHM of the $\left[\mathrm{S}_{\mathrm{II}}\right]$ profile; in the lower panel, the same as the middle panel, except that for some sources, i.e. F11394+0108 and F17234+6228, their velocity dispersions are estimated from the FWHM of the [O III] NL core because the FWHM of the $\left[\mathrm{O}_{\mathrm{III}}\right]$ NL core is smaller than that of the $\left[\mathrm{S}_{\mathrm{II}}\right]$ profile. The dashed line is the $M_{\mathrm{BH}}-\sigma$ relation from Tremaine et al. (2002). 

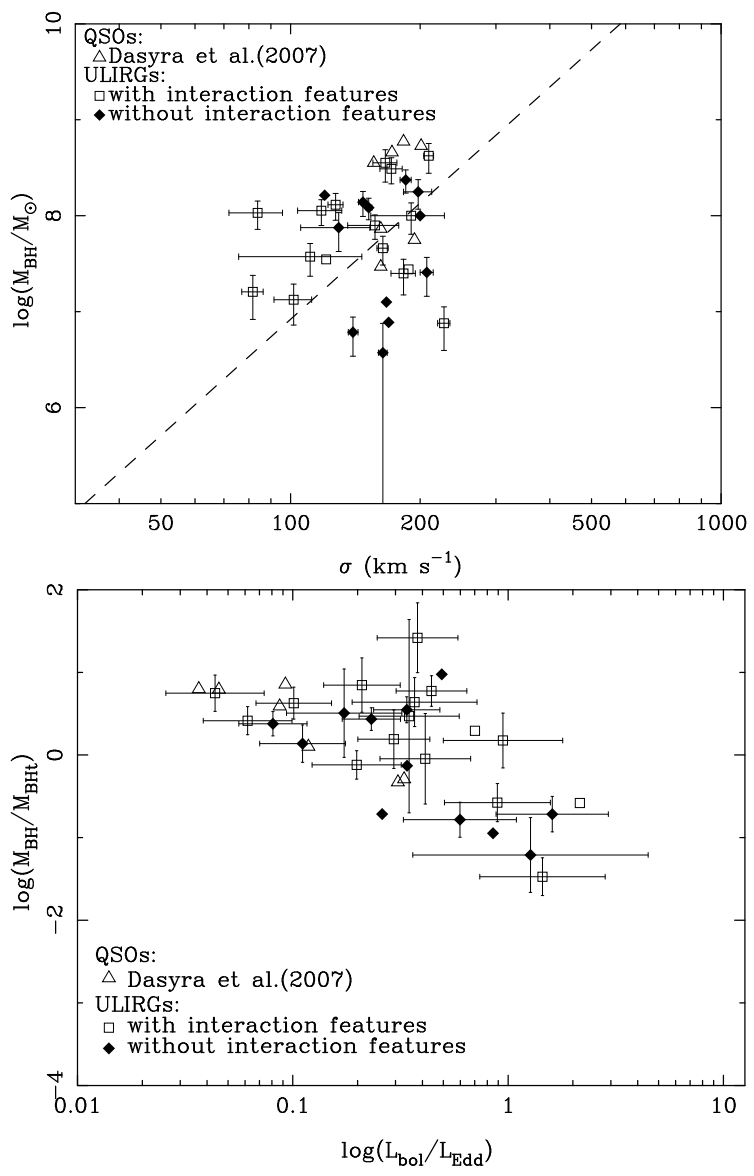

Fig. 12. $-M_{\mathrm{BH}}-\sigma$ and $\log \left(M_{\mathrm{BH}} / M_{\mathrm{BHt}}\right)$ vs. $\log \left(L_{\mathrm{bol}} / L_{\mathrm{Edd}}\right)$ diagrams for ULIRGs with or without obvious interaction features. The open squares represent the sources with interaction features, the filled rhombuses represent the ULIRGs without interaction features, and the open triangles indicate the QSOs with measured $\sigma$ in Dasyra et al. (2007). 

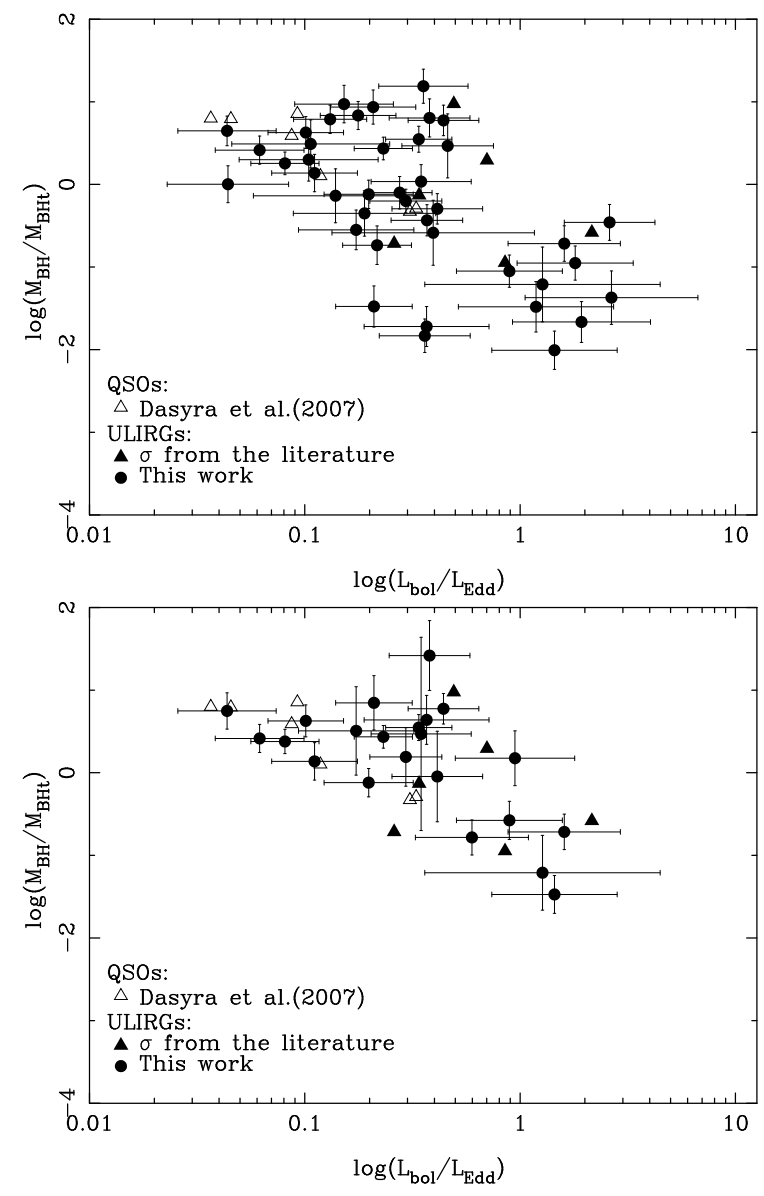

Fig. 13. $-\log \left(M_{\mathrm{BH}} / M_{\mathrm{BHt}}\right)$ vs. $\log \left(L_{\mathrm{bol}} / L_{\mathrm{Edd}}\right)$ relation of Type I ULIRGs. The filled triangles represent the six sources with measured $\sigma$ in Dasvra et al. (2006a, b, 2007), the open triangles represent the seven QSOs with measured $\sigma$ in Dasyra et al. (2007), the filled circles indicate the ULIRGs whose parameters are estimated in this work. Upper panel: the $\mathrm{M}_{\mathrm{BHt}}$ is derived from the $\sigma$ used in the upper panel of Figure 11, Lower panel: the $M_{\mathrm{BHt}}$ is obtained from the $\sigma$ used in the lower panel of Figure 11. 
middle panel, we use the FWHM of $\left[\mathrm{S}_{\mathrm{II}}\right]$ for $\sigma$ of some ULIRGs. Some ULIRGs do not follow the $M_{\mathrm{BH}}-\sigma$ relation well. The larger deviations from this relation are, i.e., F11394+0108 and F17234+6228, whose FWHMs of $\left[\mathrm{S}_{\mathrm{II}}\right]$ are broader than those of $\left[\mathrm{O}_{\mathrm{III}}\right]$ profile. The FWHM of $\left[\mathrm{O}_{\mathrm{III}}\right] \mathrm{NL}$ core is used to estimate $\sigma$ for these ULIRGs and the result is shown in the lower panel of Figure 11. Considering the error bars, we found that most ULIRGs follow the $M_{\mathrm{BH}}-\sigma$ relation, but several sources have relatively larger deviations compared with those seven QSOs. This can be seen even from those six ULIRGs with measured velocity dispersions. Therefore most Type I ULIRGs follow the $M_{\mathrm{BH}}-\sigma$ relation and probably are at a late evolutionary stage, though several ULIRGs do not follow this relation, which probably hints that AGN phase appears in the late evolutionary stage of ULIRGs, and the $M_{\mathrm{BH}}-\sigma$ relation of most Type I ULIRGs has already built up. According to the popular evolutionary picture of ULIRGs, during the evolution phase of ULIRGs to QSO, their BHs are growing, and the $M_{\mathrm{BH}}-\sigma$ relation is building up step by step. The scatter in the $M_{\mathrm{BH}}-\sigma$ diagram probably reflect the fact that ULIRGs are in different evolutionary stages and the central BHs of some ULIRGs are still rapidly growing. Therefore, the interaction properties of these Type I ULIRGs may be the hint in understanding this problem.

In our sample, F13451+3932 is an interaction system with two bright nuclei, BH mass and velocity dispersion of the west nucleus can be obtained. F10531+5531 and F15529+4545 are interaction systems. F14394+5332 interacts with a companion galaxy. Some sources show that tidal tail/plume features interact with a much smaller galaxy, such as F01572+0009, $\mathrm{F} 09591+2045$, F11162+6020, F07548+4227, F11134+0225, F14026+4341, F14315+2955, F14390+6209 F11206+3639, F11394+0108, F13342+3932, F15320+0325, F15437+4647, F16122+1531, F17234+6228. Some Type I ULIRGs are probably interacting systems but not certain because of the lower image resolution and the lack of redshift data of their companions, such as F11553-0259, F10015-0018, F14402+0108. Other ULIRGs do not show obvious interaction features at least from their SDSS images. We compare those ULIRGs with or without interaction features in the $M_{\mathrm{BH}}-\sigma$ diagram. The result is shown in Figure 12. It seems that the ULIRGs with interaction features have slightly larger deviations, but the difference is not significant. Even for those ULIRGs with interaction features, some of them are close to the $M_{\mathrm{BH}}-\sigma$ line, and others have larger deviations, which implies that the $M_{\mathrm{BH}}-\sigma$ relation for some Type I ULIRGs is not fully built up. The sources in our sample do not show large deviations from the $M_{\mathrm{BH}}-\sigma$ line, probably because the AGN phase appears in the late stage of mergers, and the interaction between central $\mathrm{BH}$ and its host galaxy already starts before the ULIRGs present broad emission lines in the optical band. More observations are needed to verify the interaction properties and merger stages of all these Type I ULIRGs. 


\subsection{Dependence on the Eddington ratio}

We show the $\log \left(M_{\mathrm{BH}} / M_{\mathrm{BHt}}\right)$ versus $\log \left(L_{\mathrm{bol}} / L_{\mathrm{Edd}}\right)$ diagram in Figure 13, where $M_{\mathrm{BHt}}$ is the BH mass calculated from the $M_{\mathrm{BH}}-\sigma$ relation of Tremaine et al. (2002). Here $\log \left(M_{\mathrm{BH}} / M_{\mathrm{BHt}}\right)$ represents the deviation of $\mathrm{BH}$ mass from the $M_{\mathrm{BH}}-\sigma$ relation. The Eddington ratio $\log \left(L_{\mathrm{bol}} / L_{\mathrm{Edd}}\right)$ usually measures the accretion rate of $\mathrm{BH}$. The uncertainty of $\log \left(M_{\mathrm{BH}} / M_{\mathrm{BHt}}\right)$ is estimated from the uncertainties of $M_{\mathrm{BH}}$, the $M_{\mathrm{BH}}-\sigma$ relation of Tremaine et al. (2002), and $\sigma$. The uncertainty of $\log \left(L_{\mathrm{bol}} / L_{\mathrm{Edd}}\right)$ is estimated from the errors of $M_{\mathrm{BH}}$ and $f_{5100 \mathrm{~A}}$. An anti-correlation trend appears in this plot even using different methods to estimate $\sigma$. For the sources with larger deviations from the $M_{\mathrm{BH}}-\sigma$ relation, they tend to have larger Eddington ratios and smaller BH masses, implying that their BHs grow faster. For the sources that are close to the $M_{\mathrm{BH}}-\sigma$ relation, they have relatively smaller Eddington ratios and larger BH masses, and their central BHs grow slowly. These results imply that different kinds of ULIRGs probably exist (i.e., some are close to the $M_{\mathrm{BH}}-\sigma$ relation and with a relatively smaller Eddington ratio, some with larger deviations to the $M_{\mathrm{BH}}-\sigma$ relation and a relatively larger Eddington ratio), and they may have evolutionary connection. One possible explanation of this trend is that the merger of gas-rich galaxies will first form a non-regular host galaxy with a larger deviation from the $M_{\mathrm{BH}}-\sigma$ relation. The interaction between central $\mathrm{BH}$ and its host galaxy will slowly make it close to the $M_{\mathrm{BH}}-\sigma$ relation, since the Eddington ratio becomes smaller, the central $\mathrm{BH}$ becomes larger and the stars around the central region form a more regular spheroid step by step. In Figure 12, we also plot the $\log \left(M_{\mathrm{BH}} / M_{\mathrm{BHt}}\right)$ versus $\log \left(L_{\mathrm{bol}} / L_{\mathrm{Edd}}\right)$ relation for ULIRGs with or without obvious interaction features.

To estimate $M_{\mathrm{BH}}$ and $L_{\mathrm{bol}} / L_{\mathrm{Edd}}$, the continuum flux at $5100 \AA$ was used. One may worry that the trend appeared in the $\log \left(M_{\mathrm{BH}} / M_{\mathrm{BHt}}\right)$ versus $\log \left(L_{\mathrm{bol}} / L_{\mathrm{Edd}}\right)$ plot is due to the common dependence of these two quantities on the continuum flux at $5100 \AA$. Because $\log \left(M_{\mathrm{BH}} / M_{\mathrm{BHt}}\right)$ is proportional to $\log \left(L_{5100 \mathrm{~A}}^{0.61}\right)$ (we used the $R-L$ relation of Kaspi et al. $2000)$, and $\log \left(L_{\mathrm{bol}} / L_{\mathrm{Edd}}\right)$ is proportional to $\log \left(L_{5100 \mathrm{~A}}^{0.39}\right)$. If the relation depends on the methods of estimating $M_{\mathrm{BH}}$ and $L_{\mathrm{bol}} / L_{\mathrm{Edd}}, \log \left(M_{\mathrm{BH}} / M_{\mathrm{BHt}}\right)$ should increase as $\log \left(\mathrm{L}_{\mathrm{bol}} / \mathrm{L}_{\mathrm{Edd}}\right)$ increases. However, this is not the case in our plot. Therefore, we believe that the anticorrelation trend between $\log \left(M_{\mathrm{BH}} / M_{\mathrm{BHt}}\right)$ and $\log \left(L_{\mathrm{bol}} / L_{\mathrm{Edd}}\right)$ is real.

Another important issue is the identification reliability of our Type I ULIRG sample. For 41 of 62 Type I ULIRGs, their counterparts and redshifts listed in the NED are consistent with our results. For other 21 ULIRGs, no counterparts and redshifts are available in the NED. As discussed in Section 2.3 and also see Figure 2, 18 of these 21 ULIRGs have their reliability greater than $93 \%$. Because the large position error of IRAS measurement, and the incompleteness of SDSS spectra, several ULIRGs identified in this work may be problematic. 
This will not affect the statistical results about the NL ULIRGs. But for Type I ULIRGs, we use 41 ULIRGs which are listed in the NED and six ULIRGs from the literature (Dasyra et al. 2006a, b, 2007) to re-do the same work, and found that our results do not change, as shown in Figure 14 .

\subsection{The relation between $L_{\mathrm{IR}}$ and $\lambda L_{\lambda}(5100 \AA)$}

The relation between IR and optical continuum luminosities is important in understanding the origin of the IR emissions in galaxies. Here we compare the samples of Hao et al. (2005) with our Type I ULIRGs, because they used the same cosmology as ours, although their calculation of $L_{\mathrm{IR}}$ is not consistent with ours (but the difference is very small, about $2 \%)$. We show the $L_{\mathrm{IR}}$ versus $\lambda L_{\lambda}(5100 \AA)$ diagram in Figure 15, There is a tight correlation between the $L_{\mathrm{IR}}$ and $\lambda L_{\lambda}(5100 \AA)$ for PG QSOs and NL Seyfert 1 galaxies. The distribution of our 62 Type I ULIRGs is consistent with that of Hao et al. (2005), and most Type I ULIRGs are above the trend which was defined by the PG QSOs and narrow line Seyfert 1 galaxies. If this correlation can be explained as both the infrared and optical continuum emissions of PG QSOs and NL Seyfert 1 are mainly from the AGN, the deviation to this correlation may imply an additional contribution to the $L_{\mathrm{IR}}$ besides the AGN in Type I ULIRGs, i.e., the contribution from starbursts.

\section{Discussions}

The intrinsic extinction of ULIRGs is significant. Kawakatu et al. (2006) estimated the optical extinction of three Type I ULIRGs using the X-ray data, and concluded that the optical extinction $A_{V}$ is probably less than 1 . While the typical optical extinction estimated from the Balmer decrement method for the NL ULIRGs is about 3 (see the $E(B-V)$ values provided in Veilleux et al. 1999a, under the assumption of $\left.R=A_{V} / E(B-V)=3.1\right)$. This result proves that the optical extinction of Type I ULIRGs is typically smaller than that of NL ULIRGs. We compared the optical extinction obtained from the X-ray method and the Balmer decrement method for these three Type I ULIRGs. For F11598-0112, the optical extinctions derived by these two methods are consistent with each other, both being about 0.03. For F01572+0009, these two methods give smaller values of the optical extinction. For the remaining one, F11119+3257, the extinctions derived by these two methods have a large difference. The extinction effect in different evolutionary stages may have a large difference. 

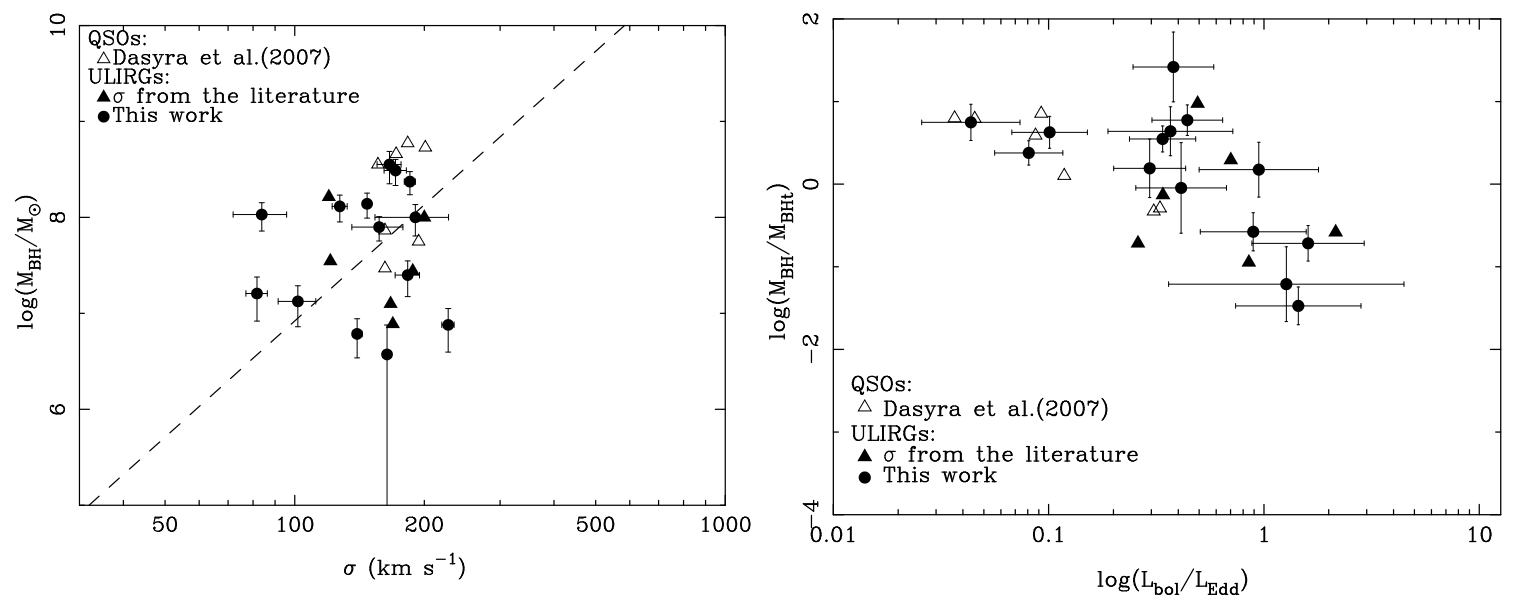

Fig. 14. - Two examples about the same results in Figures 11 and 13, but only the data have been confirmed in previous works were used.

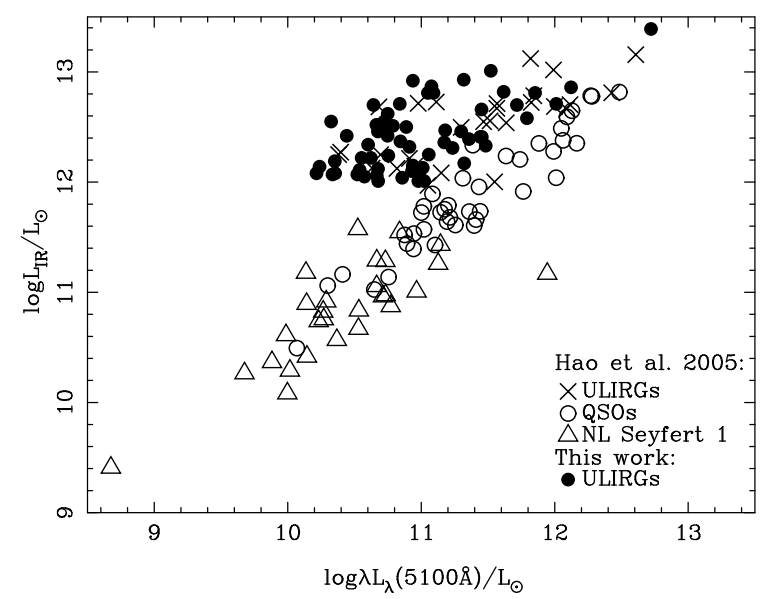

Fig. 15. - $L_{\mathrm{IR}}$ vs. $\lambda L_{\lambda}(5100 \AA)$. The open circles indicate the PG QSOs, the open triangles represent the NL Seyfert 1 galaxies, and the crosses denote the Type I ULIRGs. These data are taken from Hao et al. (2005). The filled circles are new data of the Type I ULIRGs in our sample. 
Recently, Dong et al. (2008) carried out a study on the broad-line Balmer decrement in Seyfert 1 galaxies and QSOs, and concluded that the Balmer decrement is a good indicator for dust extinction. We use the Balmer decrement method to test the influence of extinction on our results. After such a correction, the mean BH mass of our Type I ULIRGs sample becomes $\log \left(M_{\mathrm{BH}} / M_{\odot}\right) \approx 8.1$, which is larger than $\log \left(M_{\mathrm{BH}} / M_{\odot}\right) \approx 7.8$ obtained without this correction, and is still typically smaller than that of PG QSOs $\left(\log \left(M_{\mathrm{BH}} / M_{\odot}\right) \approx 8.4\right)$. The anti-correlation trend appeared in the $\log \left(M_{\mathrm{BH}} / M_{\mathrm{BHt}}\right)$ versus $\log \left(L_{\mathrm{bol}} / L_{\mathrm{Edd}}\right)$ plot still exists. Therefore, we believe that the optical extinction does not affect our main results significantly.

In Figure 11 and Figure [13, we note that the sources which are close to the $M_{\mathrm{BH}}-\sigma$ relation tend to have relatively smaller Eddington ratios and larger BH masses. The distributions of their $\mathrm{BH}$ masses and Eddington ratios are similar with those of PG QSOs (see the upper panel in Figure 13). For the ULIRGs with larger deviations from the $M_{\mathrm{BH}}-\sigma$ line, they tend to have larger Eddington ratios and smaller BH masses. An evolutionary connection may exist between them. When the BH activity in ULIRGs is active enough, the central BH can blow away a large fraction of the surrounding gases, and the ULIRGs appear as Type I ULIRGs, their central BHs are still experiencing fast growth (i.e., with a large value of Eddington ratio), and they have relatively larger deviations from the typical $M_{\mathrm{BH}}-\sigma$ relation. As the co-evolution of the central $\mathrm{BH}$ and its host galaxy, the host galaxy will form a spheroid gradually, and the growth of central BH becomes slower. At this time, the ULIRGs probably appear like optically bright QSOs. Thus the evolution of Type I ULIRGs is probably followed by the building up of the $M_{\mathrm{BH}}-\sigma$ relation and evolving towards the QSO phase.

Cao et al. (2008) compared the mid-infrared spectroscopic properties of 19 local Type I ULIRGs (named IR QSOs in their work) with that of QSOs and ULIRGs. They concluded that the MIR spectra slopes, the polycyclic aromatic hydrocarbon (PAH) emission strengths, and $\left[\mathrm{Ne}_{\mathrm{II}}\right] 12.81 \mu \mathrm{m}$ luminosities of Type I ULIRGs differ from those of PG QSOs but are comparable with ULIRGs. Their results support that Type I ULIRGs are at a transitional stage from ULIRGs to classical QSOs, which are consistent with ours. Therefore, Type I ULIRGs are important for the understanding of co-evolution of the central $\mathrm{BH}$ and its host galaxy.

\section{Conclusions}

We identified 308 ULIRGs from the SDSS DR6 spectroscopy catalog and the IRAS Faint Source Catalog. This is, to date, the largest ULIRG sample with optical spectra in the local 
universe. About $56 \%$ of them show obvious interaction features, and this percentage increases for objects with smaller redshifts, probably due to the selection effect. After excluding the ULIRGs without obvious emission lines (with $\mathrm{S} / \mathrm{N}<3$ or suffer serious absorptions around the emission lines), we obtained 147 NL ULIRGs (with only narrow emission lines in their spectra) and 62 Type I ULIRGs (with broad emission lines) in this sample. At least $49 \%$ of these 209 ULIRGs contain AGNs, and this percentage increases with $\mathrm{L}_{\mathrm{IR}}$. The ULIRGs near the radio-FIR relation mainly have the starburst related emissions, and the radio excess in some objects is due to the AGN related radio emissions.

In combination with previous data, a large sample of 90 Type I ULIRGs is used to study their $\mathrm{BH}$ masses and $\mathrm{BH}$-host galaxy relation. We found that their $\mathrm{BH}$ masses are systematically smaller than those of PG QSOs, even though the optical extinction effects were corrected through the Balmer decrement method. Their Eddington ratios are systematically larger than those of PG QSOs. Most Type I ULIRGs in our sample follow the $M_{\mathrm{BH}}-\sigma$ relation, but some do not, even the FWHM of $\left[\mathrm{O}_{\mathrm{III}}\right]$ narrow line core or $\left[\mathrm{S}_{\mathrm{II}}\right]$ was used as the surrogate of velocity dispersion, which implies that some Type I ULIRGs are in the early stage of galaxy evolution and their $M_{\mathrm{BH}}-\sigma$ relation is still building up.

We found an anti-correlation trend between $\log \left(M_{\mathrm{BH}} / M_{\mathrm{BHt}}\right)$ and $\log \left(L_{\mathrm{bol}} / L_{\mathrm{Edd}}\right)$, where $M_{\mathrm{BHt}}$ is the $\mathrm{BH}$ mass derived from the $M_{\mathrm{BH}}-\sigma$ relation of Tremaine et al. (2002). The Type I ULIRGs with larger deviations from the $M_{\mathrm{BH}}-\sigma$ relation tend to have larger Eddington ratios and smaller $\mathrm{BH}$ masses, and the ULIRGs which are close to the $M_{\mathrm{BH}}-\sigma$ relation tend to have smaller Eddington ratios and larger BH masses. This anti-correlation trend implies that the evolution of Type I ULIRGs is probably followed by the building up of the $M_{\mathrm{BH}}-\sigma$ relation and evolving towards the QSO phase. Different types of ULIRGs are probably at different evolution stages.

We thank the referee for instructive comments, and Xiaoyang Xia, Fukun Liu, Hong Wu, Minzhi Kong, Ran Wang, Zhonglue Wen, Hui Shi, and Zhaoyu Li for their helpful suggestions and discussions. This work is supported by the National Natural Science Foundation of China (10525313, 10773016,10821061 and 10833003), and the National Key Basic Research Science Foundation of China (2007CB815403, 2007CB815405). Funding for the SDSS has been provided by the Alfred P. Sloan Foundation, the Participating Institutions, the National Science Foundation, the Department of Energy, the National Aeronautics and Space Administration, the Japanese Monbukagakusho, the Max Planck Society, and the Higher Education Funding Council for England. The SDSS Web site is http://www.sdss.org. The SDSS is managed by the Astrophysical Research Consortium for the Participating Institutions. The Participating Institutions are the American Museum of Natural History, Astrophysical Institute Potsdam, University of Basel, University of Cambridge, CaseWestern Reserve University, University 
of Chicago, Drexel University, Fermilab, the Institute for Advanced Study, the Japan Participation Group, Johns Hopkins University, the Joint Institute for Nuclear Astrophysics, the Kavli Institute for Particle Astrophysics and Cosmology, the Korean Scientist Group, the Chinese Academy of Sciences (LAMOST), Los Alamos National Laboratory, the Max Planck Institute for Astronomy (MPIA), the Max Planck Institute for Astrophysics (MPA), NewMexico State University, Ohio State University, University of Pittsburgh, University of Portsmouth, Princeton University, the United States Naval Observatory, and the University of Washington.

\section{REFERENCES}

Adelman-McCarthy, J. K. et al. 2008, ApJS, 175, 297

Alexander, D. M., Brandt, W. N., Smail, I., Swinbank, A. M., Bauer, F. E., Blain, A. W., Chapman, S. C., Coppin, K. E. K., Ivison, R. J., \& Menéndez-Delmestre, K. 2008, AJ, 135,1968

Baldwin, J. A., Phillips, M. M., \& Terlevich, R. 1981, PASP, 93, 5

Becker, R. H., White, R. L., \& Helfand, D. J. 1995, ApJ, 450, 559

Best, P. N., Kauffmann, G., Heckman, T. M., \& Ivezić, Ž. 2005, MNRAS, 362, 9

Boroson, T. A. 2003, ApJ, 585, 647

Boroson, T. A. \& Green, R. F. 1992, ApJS, 80, 109

Borys, C., Smail, I., Chapman, S. C., Blain, A. W., Alexander, D. M., \& Ivison, R. J. 2005, ApJ, 635, 853

Calzetti, D., Armus, L., Bohlin, R. C., Kinney, A. L., Koornneef, J., \& Storchi-Bergmann, T. 2000, ApJ, 533, 682

Canalizo, G. \& Stockton, A. 2001, ApJ, 555, 719

Cao, C., Wu, H., Wang, J.-L., Hao, C.-N., Deng, Z.-G., Xia, X.-Y., \& Zou, Z.-L. 2006, Chinese Journal of Astronomy and Astrophysics, 6, 197

Cao, C., Xia, X. Y., Wu, H., Mao, S., Hao, C. N., \& Deng, Z. G. 2008, MNRAS, 390, 336

Cardelli, J. A., Clayton, G. C., \& Mathis, J. S. 1989, ApJ, 345, 245 
Clements, D. L., Sutherland, W. J., McMahon, R. G., \& Saunders, W. 1996, MNRAS, 279, 477

Colless, M., Dalton, G., Maddox, S., Sutherland, W., Norberg, P., Cole, S., Bland-Hawthorn, J., Bridges, T., Cannon, R., Collins, C., Couch, W., Cross, N., Deeley, K., De Propris, R., Driver, S. P., Efstathiou, G., Ellis, R. S., Frenk, C. S., Glazebrook, K., Jackson, C., Lahav, O., Lewis, I., Lumsden, S., Madgwick, D., Peacock, J. A., Peterson, B. A., Price, I., Seaborne, M., \& Taylor, K. 2001, MNRAS, 328, 1039

Condon, J. J., Anderson, M. L., \& Helou, G. 1991a, ApJ, 376, 95

Condon, J. J., Cotton, W. D., Greisen, E. W., Yin, Q. F., Perley, R. A., Taylor, G. B., \& Broderick, J. J. 1998, AJ, 115, 1693

Condon, J. J., Huang, Z.-P., Yin, Q. F., \& Thuan, T. X. 1991b, ApJ, 378, 65

Cui, J., Xia, X.-Y., Deng, Z.-G., Mao, S., \& Zou, Z.-L. 2001, AJ, 122, 63

Dasyra, K. M., Tacconi, L. J., Davies, R. I., Genzel, R., Lutz, D., Naab, T., Burkert, A., Veilleux, S., \& Sanders, D. B. 2006a, ApJ, 638, 745

Dasyra, K. M., Tacconi, L. J., Davies, R. I., Genzel, R., Lutz, D., Peterson, B. M., Veilleux, S., Baker, A. J., Schweitzer, M., \& Sturm, E. 2007, ApJ, 657, 102

Dasyra, K. M., Tacconi, L. J., Davies, R. I., Naab, T., Genzel, R., Lutz, D., Sturm, E., Baker, A. J., Veilleux, S., Sanders, D. B., \& Burkert, A. 2006b, ApJ, 651, 835

Dong, X., Wang, T., Wang, J., Yuan, W., Zhou, H., Dai, H., \& Zhang, K. 2008, MNRAS, 383,581

Farrah, D., Rowan-Robinson, M., Oliver, S., Serjeant, S., Borne, K., Lawrence, A., Lucas, R. A., Bushouse, H., \& Colina, L. 2001, MNRAS, 326, 1333

Gao, Y. \& Solomon, P. M. 2004, ApJ, 606, 271

Goto, T. 2005, MNRAS, 360, 322

Grupe, D. \& Mathur, S. 2004, ApJ, 606, L41

Hao, C. N., Xia, X. Y., Mao, S., Wu, H., \& Deng, Z. G. 2005, ApJ, 625, 78

Helou, G., Khan, I. R., Malek, L., \& Boehmer, L. 1988, ApJS, 68, 151

Helou, G., Soifer, B. T., \& Rowan-Robinson, M. 1985, ApJ, 298, L7 
Hwang, H. S., Serjeant, S., Lee, M. G., Lee, K. H., \& White, G. J. 2007, MNRAS, 375, 115

Jones, D. H., Saunders, W., Colless, M., Read, M. A., Parker, Q. A., Watson, F. G., Campbell, L. A., Burkey, D., Mauch, T., Moore, L., Hartley, M., Cass, P., James, D., Russell, K., Fiegert, K., Dawe, J., Huchra, J., Jarrett, T., Lahav, O., Lucey, J., Mamon, G. A., Proust, D., Sadler, E. M., \& Wakamatsu, K.-i. 2004, MNRAS, 355, 747

Kaspi, S., Smith, P. S., Netzer, H., Maoz, D., Jannuzi, B. T., \& Giveon, U. 2000, ApJ, 533, 631

Kauffmann, G., Heckman, T. M., Tremonti, C., Brinchmann, J., Charlot, S., White, S. D. M., Ridgway, S. E., Brinkmann, J., Fukugita, M., Hall, P. B., Ivezić, Ž., Richards, G. T., \& Schneider, D. P. 2003, MNRAS, 346, 1055

Kawakatu, N., Anabuki, N., Nagao, T., Umemura, M., \& Nakagawa, T. 2006, ApJ, 637, 104

Kewley, L. J., Groves, B., Kauffmann, G., \& Heckman, T. 2006, MNRAS, 372, 961

Kim, D.-C. \& Sanders, D. B. 1998, ApJS, 119, 41

Kim, D.-C., Veilleux, S., \& Sanders, D. B. 1998, ApJ, 508, 627

Komossa, S. \& Xu, D. 2007, ApJ, 667, L33

Lonsdale, C., Conrow, T., Evans, T., Fullmer, L., Moshir, M., Chester, T., Yentis, D., MacGillivray, H., Wolstencroft, R., \& Egret, D. 1998, in IAU Symposium, Vol. 179, New Horizons from Multi-Wavelength Sky Surveys, ed. B. J. McLean, D. A. Golombek, J. J. E. Hayes, \& H. E. Payne, 450-+

Lonsdale, C. J., Farrah, D., \& Smith, H. E. 2006, Ultraluminous Infrared Galaxies (Astrophysics Update 2), 285-+

Masci, F. J., Condon, J. J., Barlow, T. A., Lonsdale, C. J., Xu, C., Shupe, D. L., Pevunova, O., Fang, F., \& Cutri, R. 2001, PASP, 113, 10

McLure, R. J. \& Jarvis, M. J. 2002, MNRAS, 337, 109

Mirabel, I. F. \& Sanders, D. B. 1988, ApJ, 335, 104

Moshir, M., Kopman, G., \& Conrow, T. A. O. 1992, IRAS Faint Source Survey, Explanatory supplement version 2 (Pasadena: Infrared Processing and Analysis Center, California Institute of Technology, 1992, edited by Moshir, M.; Kopman, G.; Conrow, T. a.o.) 
Murphy, Jr., T. W., Armus, L., Matthews, K., Soifer, B. T., Mazzarella, J. M., Shupe, D. L., Strauss, M. A., \& Neugebauer, G. 1996, AJ, 111, 1025

Nelson, C. H. 2000, ApJ, 544, L91

Pasquali, A., Kauffmann, G., \& Heckman, T. M. 2005, MNRAS, 361, 1121

Peterson, B. M. \& Wandel, A. 1999, ApJ, 521, L95

-. 2000, ApJ, 540, L13

Roy, A. L. \& Norris, R. P. 1997, MNRAS, 289, 824

Salviander, S., Shields, G. A., Gebhardt, K., \& Bonning, E. W. 2007, ApJ, 662, 131

Sanders, D. B., Mazzarella, J. M., Kim, D.-C., Surace, J. A., \& Soifer, B. T. 2003, AJ, 126, 1607

Sanders, D. B. \& Mirabel, I. F. 1996, ARA\&A, 34, 749

Sanders, D. B., Scoville, N. Z., \& Soifer, B. T. 1991, ApJ, 370, 158

Sanders, D. B., Scoville, N. Z., Young, J. S., Soifer, B. T., Schloerb, F. P., Rice, W. L., \& Danielson, G. E. 1986, ApJ, 305, L45

Soifer, B. T., Sanders, D. B., Madore, B. F., Neugebauer, G., Danielson, G. E., Elias, J. H., Lonsdale, C. J., \& Rice, W. L. 1987, ApJ, 320, 238

Stanford, S. A., Stern, D., van Breugel, W., \& De Breuck, C. 2000, ApJS, 131, 185

Strauss, M. A., Davis, M., Yahil, A., \& Huchra, J. P. 1990, ApJ, 361, 49

Surace, J. A., Sanders, D. B., \& Evans, A. S. 2000, ApJ, 529, 170

Sutherland, W. \& Saunders, W. 1992, MNRAS, 259, 413

Tremaine, S., Gebhardt, K., Bender, R., Bower, G., Dressler, A., Faber, S. M., Filippenko, A. V., Green, R., Grillmair, C., Ho, L. C., Kormendy, J., Lauer, T. R., Magorrian, J., Pinkney, J., \& Richstone, D. 2002, ApJ, 574, 740

Veilleux, S., Kim, D.-C., \& Sanders, D. B. 1999a, ApJ, 522, 113

—. 2002, ApJS, 143, 315

Veilleux, S., Kim, D.-C., Sanders, D. B., Mazzarella, J. M., \& Soifer, B. T. 1995, ApJS, 98, 171 
Veilleux, S., Sanders, D. B., \& Kim, D.-C. 1997, ApJ, 484, 92

-. 1999b, ApJ, 522, 139

White, R. L., Becker, R. H., Helfand, D. J., \& Gregg, M. D. 1997, ApJ, 475, 479

Wu, H., Zou, Z. L., Xia, X. Y., \& Deng, Z. G. 1998a, A\&AS, 127, 521

-. 1998b, A\&AS, 132, 181

Yun, M. S., Reddy, N. A., \& Condon, J. J. 2001, ApJ, 554, 803

Zheng, X. Z., Xia, X. Y., Mao, S., Wu, H., \& Deng, Z. G. 2002, AJ, 124, 18

Zheng, Z., Wu, H., Mao, S., Xia, X.-Y., Deng, Z.-G., \& Zou, Z.-L. 1999, A\&A, 349, 735

Zou, Z., Xia, X., Deng, Z., \& Su, H. 1991, MNRAS, 252, 593

\section{A. The parameters of our samples (online version)}


Table 1. The parameters of our ULIRG sample.

\begin{tabular}{|c|c|c|c|c|c|c|c|c|c|c|c|c|c|c|}
\hline IRAS Name & $z_{\mathrm{sdss}}$ & SDSS Name & $\log \left(\frac{L_{\mathrm{IR}}}{L_{\odot}}\right)$ & $\begin{array}{c}S_{1.4 \mathrm{GHS}}^{\mathrm{NVSS}} \\
(\mathrm{mJy})\end{array}$ & $\begin{array}{c}S_{1.4 \mathrm{GHz}}^{\mathrm{FIRST}} \\
(\mathrm{mJy})\end{array}$ & Note & NED & $f_{\mathrm{H}_{\beta}}$ & $f_{\mathrm{O}_{\mathrm{III}} 5007}$ & $f_{\mathrm{H}_{\alpha}}$ & $f_{\mathrm{N}_{\mathrm{II}} 6583}$ & $f_{\mathrm{S}_{\mathrm{II}}}$ & $f_{\mathrm{O}_{\mathrm{I}} 6300}$ & Note \\
\hline (1) & (2) & (3) & (4) & $(5)$ & (6) & $(7)$ & $(8)$ & (9) & (10) & (11) & $(12)$ & (13) & (14) & $(15)$ \\
\hline
\end{tabular}

F00090-0054 0.195 J001138.79-003813.7 12.10 F01093-1002 0.131 J011152.64-094559.8 12.00 F01166-0844 0.118 J011907.57-082909.6 12.09 F01329+1439 0.218 J013537.56+145510.9 12.17 F01347+0042 0.198 J013720.02+005722.3 12.10 F01462+0014 0.280 J014852.58+002859.9 12.31 F01478+1254 0.147 J015028.40+130858.4 12.02 F01572+0009 0.163 J015950.25+002340.8 12.52 F02016-0108 0.334 J020412.43-005351.4 12.63 F02280-0746 0.295 J023028.69-073304.5 12.20 F02417-0043 0.200 J024417.44-003041.1 12.14 F02486-0714 0.327 J025105.28-070230.2 12.32 F03209-0806 0.166 J032322.87-075615.3 12.27 F03264-0705 0.220 J032855.39-065535.8 12.04 $\begin{array}{llll}\mathrm{F} 07391+3219 & 0.380 \mathrm{~J} 074217.08+321315.1 & 12.67\end{array}$ F07445+2435 0.208 J074737.14+242802.6 12.15 F07448+1813 0.340 J074741.95+180528.1 12.46 F07460+1728 0.167 J074855.47+172125.5 12.02 F07479+2646 0.384 J075104.19+263850.9 12.49 F07526+4858 0.349 J075622.12+484941.8 12.47 F07548+4227 0.211 J075819.69+421935.1 12.13

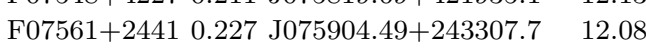
F07578+5148 0.196 J080142.16+514023.4 12.07 F07592+3736 0.238 J080234.13+372801.3 12.14 F08028+3310 0.246 J080601.71+330157.6 12.22 F08030+5243 0.083 J080650.80+523507.3 12.08 F08031+1729 0.209 J080559.47+172119.8 12.42 F08050+3909 0.368 J080824.75+390016.2 12.80 F08072+1622 0.186 J081005.28+161345.7 12.15 F08079+2822 0.350 J081107.06+281316.1 12.47 F08091+3449 0.256 J081227.65+344100.9 12.39

\begin{tabular}{|c|c|c|c|c|c|c|c|}
\hline & 1.17 & I & Yes & & & & \\
\hline \multirow[t]{3}{*}{6.2} & 5.38 & $\mathrm{n}$ & Yes & $0.92 \pm 0.07 \quad 0.61 \pm 0.04$ & $6.02 \pm 0.07$ & $3.54 \pm 0.06$ & $2.45 \pm 0.100 .46 \pm 0.03$ Composite \\
\hline & 2.10 & III & Yes & $0.73 \pm 0.04 \quad 0.15 \pm 0.03$ & $3.11 \pm 0.06$ & $1.67 \pm 0.04$ & $1.02 \pm 0.040 .06 \pm 0.02$ Starburst \\
\hline & & $\mathrm{n}$ & Yes & $6.14 \pm 0.06 \quad 13.51 \pm 0.09$ & $26.22 \pm 0.16$ & $5.03 \pm 0.06$ & $6.22 \pm 0.070 .87 \pm 0.03$ Starburst \\
\hline 3.3 & 2.92 & III & Yes & $0.32 \pm 0.10 \quad 0.20 \pm 0.04$ & $2.92 \pm 0.08$ & $2.05 \pm 0.07$ & $1.37 \pm 0.070 .25 \pm 0.04$ Composite \\
\hline \multirow[t]{2}{*}{2.8} & 2.38 & II & Yes & $0.92 \pm 0.04 \quad 0.76 \pm 0.03$ & $6.70 \pm 0.10$ & $3.82 \pm 0.09$ & $2.27 \pm 0.080 .33 \pm 0.05$ Composite \\
\hline & & III & Yes & $7.45 \pm 0.13 \quad 17.94 \pm 0.09$ & $28.73 \pm 0.13$ & $6.30 \pm 0.04$ & $6.21 \pm 0.050 .81 \pm 0.03$ Starburst \\
\hline \multirow[t]{3}{*}{26.2} & 24.08 & I & Yes & & & & \\
\hline & & $\mathrm{n}$ & Yes & & & & \\
\hline & 1.27 & $\mathrm{n}$ & Yes & $0.53 \pm 0.05 \quad 0.37 \pm 0.03$ & $4.56 \pm 0.09$ & $2.80 \pm 0.06$ & $2.08 \pm 0.080 .19 \pm 0.04$ Composite \\
\hline 10.3 & 9.39 & III & Yes & $0.64 \pm 0.05 \quad 5.77 \pm 0.46$ & $8.43 \pm 0.14$ & $15.63 \pm 0.15$ & $4.50 \pm 0.090 .97 \pm 0.06 \quad$ Seyfert \\
\hline 6.9 & 5.80 & I & Yes & & & & \\
\hline \multirow[t]{8}{*}{4.8} & & I & Yes & $0.94 \pm 0.04 \quad 0.91 \pm 0.05$ & $9.24 \pm 0.07$ & $6.92 \pm 0.06$ & $3.26 \pm 0.060 .58 \pm 0.04$ Composite \\
\hline & & II & No & & & & \\
\hline & & II & Yes & & & & \\
\hline & 1.35 & $\mathrm{n}$ & No & & & & \\
\hline & & $\mathrm{n}$ & No & & & & \\
\hline & & III & No & & & & \\
\hline & 1.62 & I & Yes & & & & \\
\hline & & $\mathrm{n}$ & Yes & & & & \\
\hline 3.4 & 1.81 & II & Yes & & & & \\
\hline \multirow[t]{4}{*}{2.5} & & II & No & & & & \\
\hline & & $\mathrm{n}$ & No & & & & \\
\hline & 3.36 & III & Yes & $1.78 \pm 0.07 \quad 6.91 \pm 0.07$ & $12.22 \pm 0.12$ & $9.84 \pm 0.10$ & $6.89 \pm 0.101 .53 \pm 0.06 \quad$ Seyfert \\
\hline & 1.93 & $\mathrm{n}$ & No & $0.85 \pm 0.04 \quad 2.61 \pm 0.05$ & $7.87 \pm 0.10$ & $4.78 \pm 0.08$ & $2.12 \pm 0.090 .47 \pm 0.06$ Ambiguity \\
\hline \multirow[t]{6}{*}{15.3} & 15.36 & $\mathrm{n}$ & Yes & $1.91 \pm 0.05 \quad 1.60 \pm 0.06$ & $24.10 \pm 0.17$ & $12.03 \pm 0.11$ & $7.73 \pm 0.111 .10 \pm 0.05$ Composite \\
\hline & & I & No & & & & \\
\hline & & $\mathrm{n}$ & No & & & & \\
\hline & 2.14 & $\mathrm{n}$ & Yes & $1.83 \pm 0.04 \quad 6.08 \pm 0.06$ & $12.18 \pm 0.11$ & $7.90 \pm 0.08$ & $3.39 \pm 0.080 .37 \pm 0.04$ Ambiguity \\
\hline & & $\mathrm{n}$ & Yes & & & & \\
\hline & 2.98 & $\mathrm{n}$ & Yes & $0.39 \pm 0.13 \quad 6.73 \pm 0.16$ & $3.82 \pm 0.08$ & $4.97 \pm 0.10$ & $2.48 \pm 0.10 \quad 0.57 \pm 0.06 \quad$ Seyfert \\
\hline
\end{tabular}


Table 1-Continued

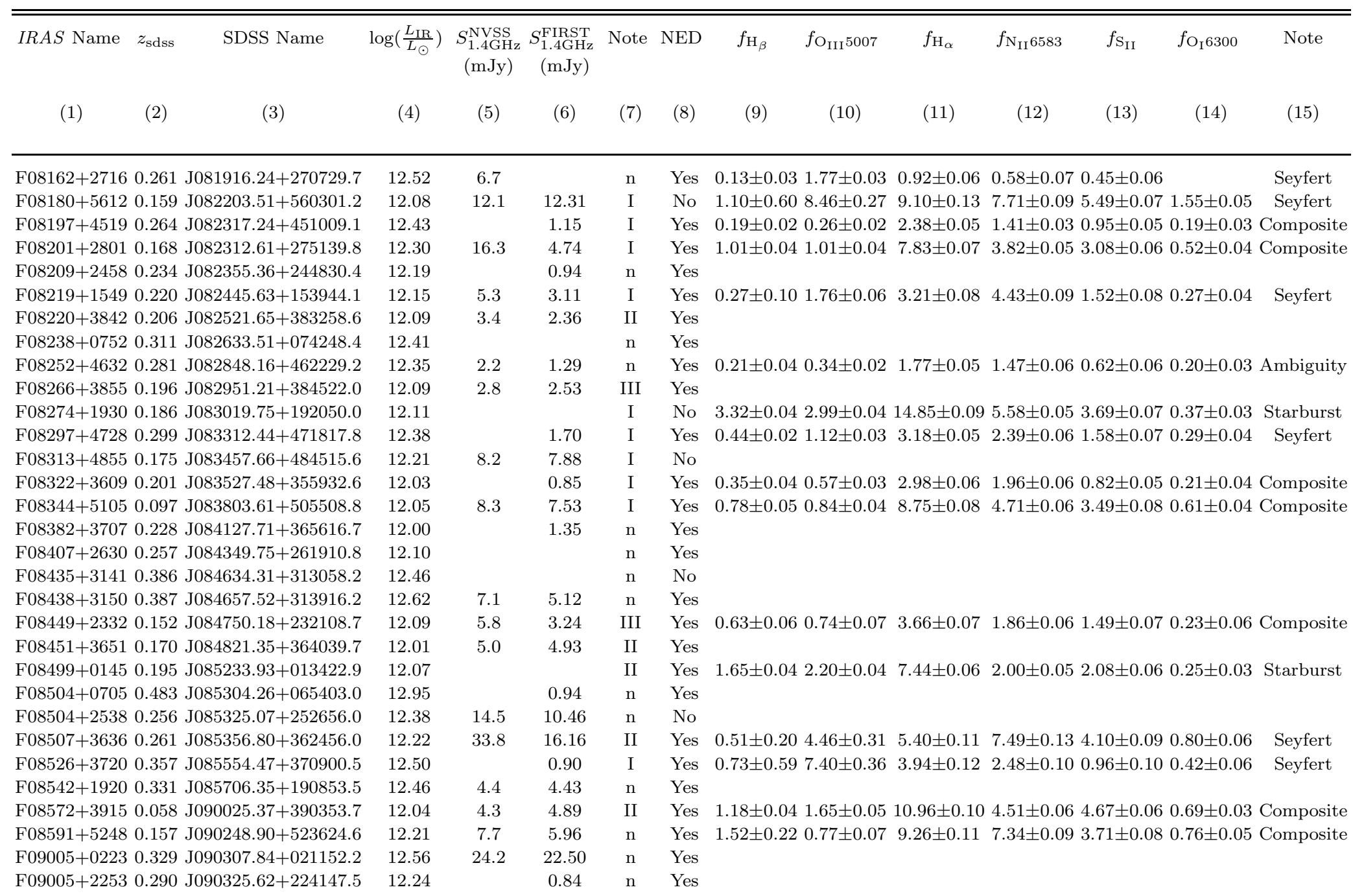


Table 1-Continued

\begin{tabular}{|c|c|c|c|c|c|c|c|c|c|c|c|c|c|}
\hline IRAS Name & SDSS Name & $\log \left(\frac{L_{\mathrm{IR}}}{L_{\odot}}\right)$ & $\begin{array}{c}S_{1.4 \mathrm{GHz}}^{\mathrm{NVSS}} \\
(\mathrm{mJy}) \\
(5)\end{array}$ & $\begin{array}{c}S_{1.4 \mathrm{GHz}}^{\mathrm{FIRST}} \\
(\mathrm{mJy}) \\
(6)\end{array}$ & Note & NED & $f_{\mathrm{H}_{\beta}}$ & $f_{\mathrm{O}_{\mathrm{III}} 5007}$ & $f_{\mathrm{H}_{\alpha}}$ & $\begin{array}{r}f_{\mathrm{N}_{\mathrm{II}} 6583} \\
(12)\end{array}$ & $f_{\mathrm{S}_{\mathrm{II}}}$ & $\begin{array}{r}f_{\mathrm{O}_{\mathrm{I}} 6300} \\
(14)\end{array}$ & Note \\
\hline F09008+3643 & 0.288 J090359.91+363054.7 & 12.55 & & 2.05 & $\mathrm{n}$ & Yes & & & & & & & \\
\hline F09025+1253 & 0.196 J090517.05+124151.9 & 12.17 & 4.2 & & II & No & & & & & & & \\
\hline F09029+2430 & 0.232 J090554.71+241828.8 & 12.15 & 3.9 & 3.48 & II & No & $0.07 \pm 0.03$ & $0.78 \pm 0.05$ & $2.35 \pm 0.08$ & $3.62 \pm 0.09$ & $2.15 \pm 0.10$ & $0.52 \pm 0.06$ & Seyfert \\
\hline F09036+2706 & 0.341 J090633.96+265419.6 & 12.39 & & 1.44 & $\mathrm{n}$ & No & $0.95 \pm 0.05$ & $3.72 \pm 0.04$ & $5.39 \pm 0.10$ & $1.82 \pm 0.15$ & $0.97 \pm 0.09$ & $0.23 \pm 0.03$ & Ambiguity \\
\hline F09039+0503 & 0.125 J090634.04+045127.6 & 12.14 & 6.2 & 4.92 & I & Yes & $1.63 \pm 0.06$ & $1.42 \pm 0.06$ & $11.71 \pm 0.11$ & $10.50 \pm 0.10$ & $6.72 \pm 0.08$ & $1.43 \pm 0.05$ & Composite \\
\hline F09045+3943 & 0.224 J090742.25+393149.8 & 12.02 & & 1.24 & I & Yes & $0.82 \pm 0.04$ & $1.15 \pm 0.03$ & $4.17 \pm 0.06$ & $1.56 \pm 0.05$ & $1.60 \pm 0.06$ & $0.28 \pm 0.04$ & Composite \\
\hline F09083+0311 & 0.504 J091050.01+025939.6 & 13.00 & 6.1 & 4.26 & $\mathrm{n}$ & Yes & & & & & & & \\
\hline F09097+5342 & 0.254 J091319.02+532958.5 & 12.07 & & 0.87 & III & No & $0.32 \pm 0.04$ & $1.20 \pm 0.05$ & $3.48 \pm 0.09$ & $3.87 \pm 0.08$ & $1.96 \pm 0.08$ & $0.34 \pm 0.04$ & Seyfert \\
\hline F09105+4108 & 0.442 J091345.49+405628.2 & 12.87 & 15.9 & 8.27 & $\mathrm{I}$ & Yes & & & & & & & \\
\hline F09116+0334 & 0.145 J091413.79+032201.3 & 12.18 & 10.5 & 9.41 & I & Yes & $1.48 \pm 0.45$ & $0.72 \pm 0.08$ & $12.11 \pm 0.14$ & $11.67 \pm 0.13$ & $4.44 \pm 0.12$ & $0.71 \pm 0.07$ & Composite \\
\hline F09119+0945 & 0.246 J091438.14+093322.9 & 12.31 & & 1.18 & II & Yes & $0.48 \pm 0.03$ & $0.40 \pm 0.03$ & $3.47 \pm 0.05$ & $3.19 \pm 0.05$ & $1.02 \pm 0.06$ & $0.24 \pm 0.06$ & Composite ! \\
\hline F09186+4521 & 0.235 J092159.39+450912.4 & 12.04 & & & $\mathrm{n}$ & No & $1.97 \pm 0.06$ & $1.91 \pm 0.06$ & $10.89 \pm 0.11$ & $5.56 \pm 0.08$ & $2.99 \pm 0.09$ & $0.62 \pm 0.05$ & Composite \\
\hline F09198+0323 & 0.174 J092226.71+031048.2 & 12.05 & 2.2 & & II & Yes & & & & & & & \\
\hline F09217+5348 & 0.195 J092518.47+533525.0 & 12.04 & & 1.20 & $\mathrm{I}$ & No & $0.24 \pm 0.11$ & $0.18 \pm 0.03$ & $1.18 \pm 0.04$ & $1.39 \pm 0.05$ & $0.93 \pm 0.05$ & $0.22 \pm 0.03$ & LINERs \\
\hline F09220+2759 & 0.531 J092501.79+274608.0 & 12.92 & 26.3 & 26.74 & $\mathrm{n}$ & No & & & & & & & \\
\hline F09240+4804 & 0.237 J092723.13+475147.3 & 12.07 & & & II & No & $1.12 \pm 0.03$ & $0.54 \pm 0.03$ & $6.48 \pm 0.06$ & $2.94 \pm 0.05$ & $1.56 \pm 0.06$ & & Starburst \\
\hline F09246+0115 & 0.169 J092710.88+010232.2 & 12.07 & & 2.66 & I & Yes & $1.32 \pm 0.05$ & $0.61 \pm 0.05$ & $10.75 \pm 0.08$ & $5.00 \pm 0.05$ & $3.28 \pm 0.08$ & $0.46 \pm 0.04$ & Starburst \\
\hline F09258+1735 & 0.252 J092838.64+172220.4 & 12.16 & 5.6 & 5.76 & $\mathrm{n}$ & No & & & & & & & \\
\hline F09306+5431 & 0.232 J093402.47+541751.5 & 12.17 & & & III & Yes & & & & & & & \\
\hline F09320+6134 & 0.039 J093551.61+612111.4 & 12.02 & 170.1 & 146.74 & $\mathrm{I}$ & Yes & $1.29 \pm 0.07$ & $2.56 \pm 0.07$ & $22.31 \pm 0.17$ & $29.70 \pm 0.18$ & $9.19 \pm 0.12$ & $1.60 \pm 0.07$ & Ambiguity \\
\hline F09322+0432 & 0.198 J093451.87+041848.2 & 12.13 & 3.0 & 1.41 & II & Yes & & & & & & & \\
\hline $\mathrm{F} 09323+6222$ & 0.225 J093613.72+620905.4 & 12.06 & & & I & No & & & & & & & \\
\hline F09395+3939 & 0.194 J094239.17+392559.7 & 12.14 & 3.5 & 3.53 & II & Yes & $0.45 \pm 0.05$ & $0.57 \pm 0.06$ & $6.24 \pm 0.08$ & $3.62 \pm 0.09$ & $1.96 \pm 0.09$ & $0.37 \pm 0.05$ & Composite \\
\hline F09398+0013 & 0.146 J094224.28-000005.0 & 12.06 & 5.5 & 4.87 & I & Yes & $0.83 \pm 0.04$ & $1.67 \pm 0.05$ & $10.26 \pm 0.08$ & $5.52 \pm 0.06$ & $4.48 \pm 0.07$ & $0.79 \pm 0.04$ & Composite \\
\hline $\mathrm{F} 09437+1720$ & 0.243 J094635.73+170558.4 & 12.02 & & 1.97 & $\mathrm{n}$ & No & $2.30 \pm 0.04$ & $1.49 \pm 0.04$ & $11.55 \pm 0.11$ & $5.62 \pm 0.07$ & $3.81 \pm 0.10$ & $0.75 \pm 0.05$ & Composite \\
\hline $\mathrm{F} 09438+4735$ & 0.539 J094704.52+472143.0 & 13.01 & 3.1 & 2.84 & $\mathrm{n}$ & Yes & & & & & & & \\
\hline F09444+1019 & 0.202 J094706.99+100611.6 & 12.01 & & 1.97 & I & No & $1.59 \pm 0.06$ & $1.47 \pm 0.05$ & $9.29 \pm 0.10$ & $3.77 \pm 0.08$ & $3.10 \pm 0.07$ & $0.58 \pm 0.04$ & Composite Starburst \\
\hline F09469+6234 & 0.213 J095047.39+622021.3 & 12.00 & & & $\mathrm{n}$ & No & & & & & & & \\
\hline F09501+5535 & 0.324 J095331.87+552102.2 & 12.54 & 2.5 & 1.07 & $\mathrm{n}$ & Yes & & & & & & & \\
\hline F09555+5109 & 0.215 J095846.85+505456.5 & 12.18 & & & II & No & $0.47 \pm 0.11$ & $1.39 \pm 0.04$ & $3.50 \pm 0.08$ & $1.95 \pm 0.07$ & $1.25 \pm 0.06$ & $0.16 \pm 0.04$ & Seyfert \\
\hline F09583+4714 & $0.086 \mathrm{~J} 100131.21+465946.8$ & 12.02 & & 24.95 & II & Yes & $3.05 \pm 0.64$ & $11.21 \pm 0.52$ & $62.91 \pm 0.21$ & $25.23 \pm 0.96$ & $13.98 \pm 0.11$ & $5.09 \pm 0.07$ & Composite \\
\hline
\end{tabular}


Table 1-Continued

\begin{tabular}{|c|c|c|c|c|c|c|c|c|c|c|c|c|c|c|}
\hline IRAS Name & $z_{\mathrm{sdss}}$ & SDSS Name & $\log \left(\frac{L_{\mathrm{IR}}}{L_{\odot}}\right)$ & $\begin{array}{c}S_{1.4 \mathrm{GVS}}^{\mathrm{NVS}} \\
(\mathrm{mJy})\end{array}$ & $\begin{array}{c}S_{1.4 \mathrm{GHz}}^{\mathrm{FIRST}} \\
(\mathrm{mJy})\end{array}$ & Note & NED & $f_{\mathrm{H}_{\beta}}$ & $f_{\mathrm{O}_{\mathrm{III}} 5007}$ & $f_{\mathrm{H}_{\alpha}}$ & $f_{\mathrm{N}_{\text {II }} 6583}$ & $f_{\mathrm{S}_{\mathrm{II}}}$ & $f_{\mathrm{O}_{\mathrm{I}} 6300}$ & Note \\
\hline (1) & (2) & (3) & (4) & (5) & (6) & $(7)$ & (8) & (9) & $(10)$ & (11) & (12) & (13) & (14) & $(15)$ \\
\hline
\end{tabular}

\begin{tabular}{|c|c|c|c|c|c|c|c|c|c|c|c|}
\hline F09591+2045 0.357 J100153.66+203141.1 & 12.50 & & 1.35 & I & No & & & & & & \\
\hline F10015-0018 0.289 J100404.99-003253.4 & 12.52 & 4.0 & 2.55 & I & Yes & & & & & & \\
\hline F10017+4011 0.312 J100445.17+395732.3 & 12.27 & & 1.61 & $\mathrm{n}$ & Yes & & & & & & \\
\hline F10022+5233 0.226 J100539.16+521842.7 & 12.05 & & & $\mathrm{n}$ & No & & & & & & \\
\hline F10026+3107 0.235 J100532.13+305318.2 & 12.03 & 2.5 & 3.62 & $\mathrm{n}$ & Yes & & & & & & \\
\hline F10026+4347 0.179 J100541.86+433240.3 & 12.08 & & 2.81 & $\mathrm{n}$ & Yes & & & & & & \\
\hline F10026-0022 0.407 J100513.11-003721.4 & 12.66 & & & $\mathrm{n}$ & No & & & & & & \\
\hline F10030+4126 0.328 J100603.85+411224.8 & 12.49 & & 2.21 & $\mathrm{n}$ & Yes & $0.86 \pm 0.03$ & $1.57 \pm 0.04$ & $5.23 \pm 0.10$ & $1.60 \pm 0.08$ & $1.94 \pm 0.18$ & Starburst \\
\hline F10035+2740 0.166 J100626.33+272546.4 & 12.30 & 5.8 & 5.44 & I & Yes & $0.32 \pm 0.04$ & $0.66 \pm 0.05$ & $2.72 \pm 0.06$ & $2.91 \pm 0.05$ & $2.46 \pm 0.06 \quad 0.52 \pm 0.04$ & LINERs \\
\hline F10035+4852 0.065 J100645.86+483743.8 & 12.03 & 28.1 & 8.59 & II & Yes & $1.00 \pm 0.05$ & $0.41 \pm 0.04$ & $7.81 \pm 0.08$ & $3.97 \pm 0.06$ & $3.04 \pm 0.06 \quad 0.35 \pm 0.03$ & Composite \\
\hline F10037+1112 0.274 J100624.37+105748.4 & 12.15 & & 1.15 & $\mathrm{n}$ & Yes & $1.58 \pm 0.04$ & $4.06 \pm 0.05$ & $8.05 \pm 0.09$ & $2.22 \pm 0.06$ & $2.27 \pm 0.08 \quad 0.37 \pm 0.04$ & Composite \\
\hline F10040+0932 0.171 J100643.50+091727.5 & 12.22 & 3.0 & 2.58 & $\mathrm{n}$ & Yes & $1.16 \pm 0.07$ & $0.86 \pm 0.05$ & $7.28 \pm 0.07$ & $3.87 \pm 0.07$ & $2.37 \pm 0.070 .35 \pm 0.04$ & Composite \\
\hline F10052+2959 0.257 J100809.48+294425.9 & 12.11 & 2.6 & 1.66 & $\mathrm{n}$ & Yes & $0.22 \pm 0.10$ & $0.49 \pm 0.06$ & $2.51 \pm 0.08$ & $3.05 \pm 0.10$ & $0.87 \pm 0.10$ & Ambiguity \\
\hline F10059+3605 0.161 J100852.90+355103.0 & 12.03 & & & $\mathrm{n}$ & No & & & & & & \\
\hline F10105+6118 0.213 J101358.92+610431.9 & 12.01 & & 0.85 & I & No & $1.17 \pm 0.07$ & $1.49 \pm 0.04$ & $5.64 \pm 0.08$ & $2.41 \pm 0.06$ & $1.85 \pm 0.06 \quad 0.33 \pm 0.04$ & Composite \\
\hline F10106+2227 0.274 J101325.42+221229.4 & 12.15 & & 1.08 & $\mathrm{n}$ & No & & & & & & \\
\hline F10107+4708 0.206 J101348.08+465359.5 & 12.34 & & 2.15 & I & No & & & & & & \\
\hline $\mathrm{F} 10124+2742 \quad 0.210 \mathrm{~J} 101515.35+272717.1$ & 12.24 & 5.6 & 5.36 & $\mathrm{n}$ & No & $1.68 \pm 0.07$ & $4.24 \pm 0.07$ & $12.70 \pm 0.11$ & $9.16 \pm 0.09$ & $5.13 \pm 0.070 .91 \pm 0.07$ & Seyfert \\
\hline F10190+1322 0.076 J102142.79+130656.1 & 12.07 & 16.8 & 16.41 & II & Yes & & & & & & \\
\hline F10194+2427 0.188 J102212.64+241202.4 & 12.04 & 3.9 & 4.08 & I & No & $0.51 \pm 0.15$ & $1.17 \pm 0.05$ & $4.17 \pm 0.08$ & $3.30 \pm 0.07$ & $1.55 \pm 0.070 .29 \pm 0.04$ & Ambiguity \\
\hline F10196+3707 0.267 J102229.18+365209.7 & 12.20 & & 1.42 & III & No & $1.62 \pm 0.04$ & $1.53 \pm 0.03$ & $9.02 \pm 0.09$ & $4.11 \pm 0.09$ & $2.70 \pm 0.070 .42 \pm 0.04$ & Composite \\
\hline $\mathrm{F} 10211+2436 \quad 0.209 \mathrm{~J} 102359.20+242106.3$ & 12.06 & & & I & No & $6.07 \pm 0.06$ & $16.62 \pm 0.09$ & $23.63 \pm 0.16$ & $4.92 \pm 0.07$ & $4.41 \pm 0.06 \quad 0.67 \pm 0.04$ & Starburst \\
\hline F10212+2506 0.196 J102403.67+245139.3 & 12.11 & 2.6 & 1.87 & I & Yes & & & & & & \\
\hline F10234+3052 0.340 J102617.48+303643.1 & 12.47 & 3.4 & 1.82 & $\mathrm{n}$ & Yes & & & & & & \\
\hline F10253+0854 0.417 J102755.84+083913.3 & 12.65 & & & II & Yes & & & & & & \\
\hline F10341+1312 0.174 J103650.91+125714.7 & 12.06 & 4.9 & 3.16 & $\mathrm{n}$ & Yes & & & & & & \\
\hline F10345+3809 0.203 J103728.97+375407.6 & 12.05 & & & I & No & $2.41 \pm 0.06$ & $4.22 \pm 0.05$ & $11.76 \pm 0.11$ & $2.30 \pm 0.07$ & $3.79 \pm 0.070 .52 \pm 0.04$ & Starburst \\
\hline F10360+1428 0.375 J103840.87+141307.5 & 12.51 & & & II & Yes & & & & & & \\
\hline F10369+4913 0.175 J104000.53+485744.5 & 12.06 & 7.5 & 7.25 & I & Yes & $3.01 \pm 0.06$ & $2.07 \pm 0.07$ & $15.34 \pm 0.13$ & $7.65 \pm 0.08$ & $5.14 \pm 0.090 .84 \pm 0.05$ & Composite \\
\hline F10372+4801 0.486 J104014.42+474554.7 & 12.81 & 4.1 & 4.28 & $\mathrm{n}$ & Yes & & & & & & \\
\hline F10378+1108 0.136 J104029.17+105318.3 & 12.34 & 8.4 & 8.55 & I & Yes & $1.30 \pm 0.28$ & $2.75 \pm 0.35$ & $13.63 \pm 0.13$ & $15.42 \pm 0.13$ & $8.66 \pm 0.122 .08 \pm 0.06$ & LINERs \\
\hline
\end{tabular}


Table 1-Continued

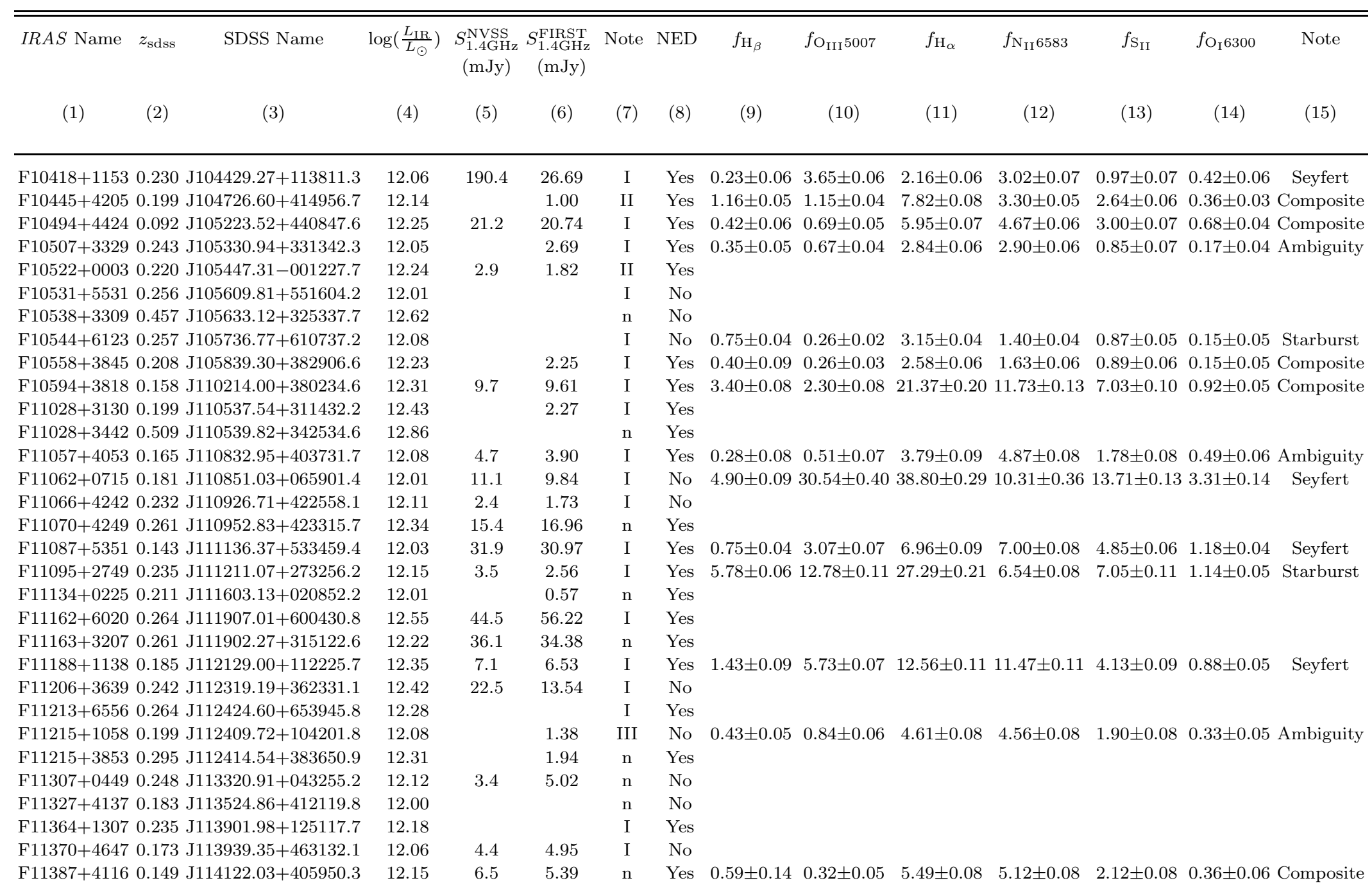


Table 1-Continued

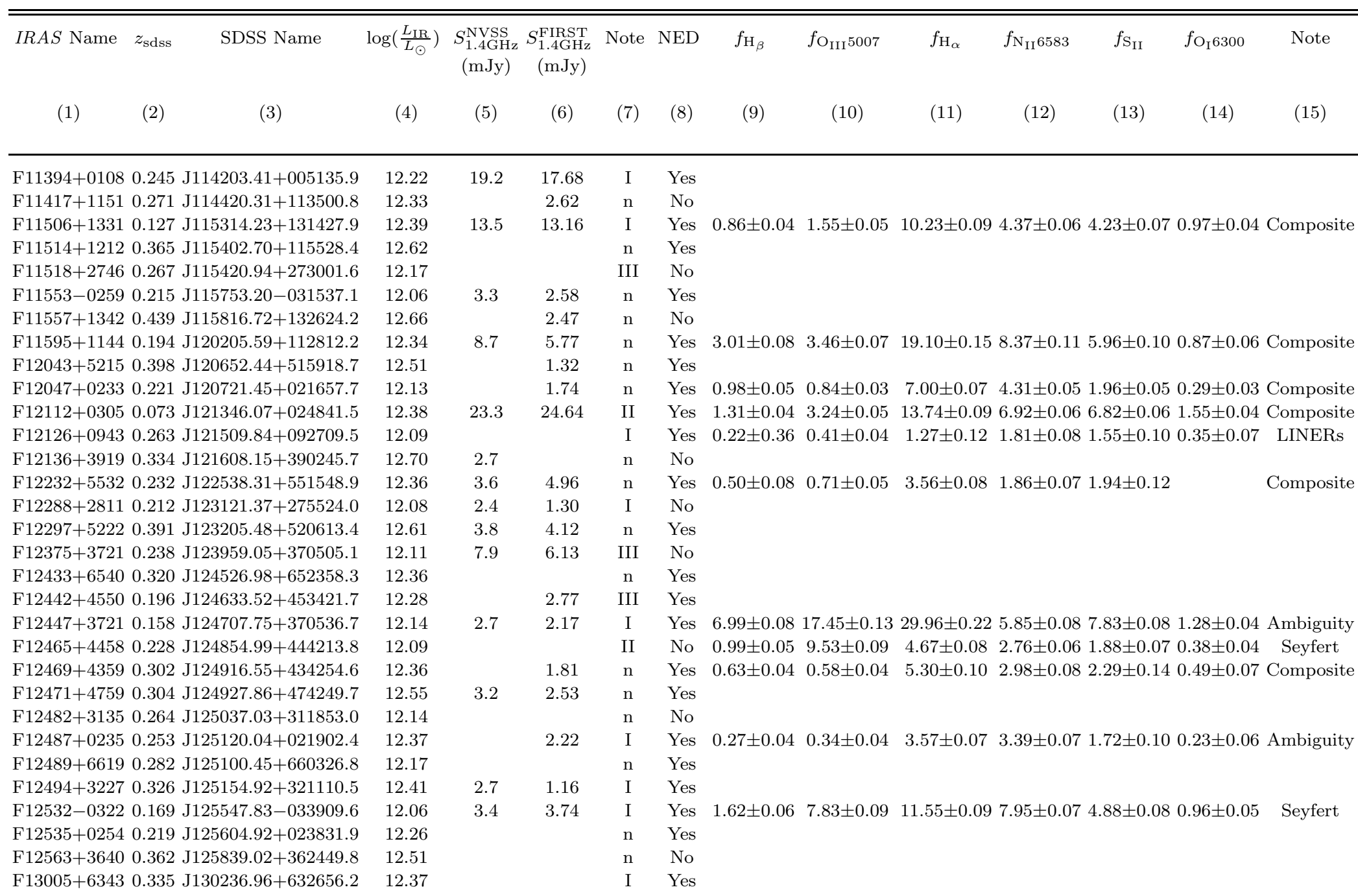


Table 1-Continued

\begin{tabular}{|c|c|c|c|c|c|c|c|c|c|c|c|c|c|c|}
\hline IRAS Name & $z_{\text {sdss }}$ & SDSS Name & $\log \left(\frac{L_{\mathrm{IR}}}{L_{\odot}}\right)$ & $\begin{array}{c}S_{1.4 \mathrm{GHz}}^{\mathrm{NVSS}} \\
(\mathrm{mJy})\end{array}$ & $\begin{array}{c}S_{1.4 \mathrm{GHz}}^{\mathrm{FIRST}} \\
(\mathrm{mJy})\end{array}$ & Note & NED & $f_{\mathrm{H}_{\beta}}$ & $f_{\mathrm{O}_{\mathrm{III}} 5007}$ & $f_{\mathrm{H}_{\alpha}}$ & $f_{\mathrm{N}_{\mathrm{II}} 6583}$ & $f_{\mathrm{S}_{\mathrm{II}}}$ & $f_{\mathrm{O}_{\mathrm{I}} 6300}$ & Note \\
\hline (1) & $(2)$ & $(3)$ & $(4)$ & $(5)$ & $(6)$ & (7) & (8) & $(9)$ & $(10)$ & (11) & $(12)$ & (13) & $(14)$ & $(15)$ \\
\hline
\end{tabular}

\begin{tabular}{|c|c|c|c|c|c|c|c|c|c|c|c|c|}
\hline F13096+4507 0.232 J131201.62+445109.9 & 12.20 & & & $\mathrm{n}$ & No & & & & & & & \\
\hline F13161+0927 0.282 J131842.18+091112.9 & 12.22 & & 0.99 & I & No & $0.89 \pm 0.06$ & $2.82 \pm 0.05$ & $7.34 \pm 0.09$ & $6.99 \pm 0.10$ & $2.57 \pm 0.10$ & $0.59 \pm 0.05$ & Seyfert \\
\hline F13163+5237 0.355 J131827.08+522049.8 & 12.66 & & & $\mathrm{n}$ & No & & & & & & & \\
\hline $\mathrm{F} 13190+4050 \quad 0.185 \mathrm{~J} 132117.48+403519.5$ & 12.08 & 2.5 & 2.62 & $\mathrm{n}$ & Yes & $0.28 \pm 0.21$ & $1.48 \pm 0.08$ & $3.60 \pm 0.09$ & $3.98 \pm 0.10$ & $1.39 \pm 0.11$ & $0.19 \pm 0.06$ & Seyfert \\
\hline F13205+3853 0.428 J132248.41+383816.5 & 12.55 & & & $\mathrm{n}$ & No & & & & & & & \\
\hline F13209+6353 $0.200 \mathrm{~J} 132244.13+633724.9$ & 12.21 & 6.8 & 6.72 & I & Yes & $0.60 \pm 0.06$ & $4.14 \pm 0.28$ & $5.07 \pm 0.11$ & $7.58 \pm 0.11$ & $3.98 \pm 0.08$ & $1.11 \pm 0.06$ & Seyfert \\
\hline F13210+3932 0.566 J132317.01+391626.5 & 12.93 & & & $\mathrm{n}$ & Yes & & & & & & & \\
\hline F13218+0552 0.203 J132419.89+053704.7 & 12.41 & 4.9 & 4.26 & I & Yes & & & & & & & \\
\hline $\mathrm{F} 13231+6235 \quad 0.237 \mathrm{~J} 132456.30+621958.1$ & 12.31 & 3.9 & 2.85 & I & Yes & & & & & & & \\
\hline $\mathrm{F} 13243+6308 \quad 0.242 \mathrm{~J} 132608.51+625207.3$ & 12.28 & & & $\mathrm{n}$ & No & $1.05 \pm 0.04$ & $1.40 \pm 0.04$ & $4.32 \pm 0.07$ & $1.23 \pm 0.05$ & $1.37 \pm 0.09$ & & Starburst \\
\hline F13342+3932 0.179 J133624.06+391731.0 & 12.37 & 6.4 & 6.94 & I & Yes & & & & & & & \\
\hline F13403-0038 0.326 J134251.61-005345.3 & 12.39 & & & $\mathrm{n}$ & Yes & & & & & & & \\
\hline F13408+4047 0.906 J134252.95+403201.6 & 13.47 & 151.6 & 136.55 & $\mathrm{n}$ & Yes & & & & & & & \\
\hline F13428+5608 0.037 J134442.16+555313.5 & 12.16 & 144.7 & 132.02 & I & Yes & $8.18 \pm 0.33$ & $31.33 \pm 1.36$ & $82.06 \pm 0.62$ & $87.51 \pm 0.56$ & $49.47 \pm 0.35$ & $10.57 \pm 0.18$ & Seyfert \\
\hline $\mathrm{F} 13446+3727 \quad 0.215 \mathrm{~J} 134651.82+371231.0$ & 12.05 & 2.6 & 2.02 & I & Yes & $2.87 \pm 0.06$ & $9.71 \pm 0.08$ & $15.36 \pm 0.13$ & $10.92 \pm 0.11$ & $4.54 \pm 0.08$ & $0.70 \pm 0.05$ & Seyfert \\
\hline $\mathrm{F} 13451+12320.121 \mathrm{~J} 134733.36+121724.3$ & 12.19 & & 4859.88 & II & Yes & & & & & & & \\
\hline F13457+5702 0.209 J134732.49+564736.7 & 12.00 & & 1.60 & II & No & & & & & & & \\
\hline F13469+5833 0.158 J134840.07+581851.9 & 12.29 & 3.1 & 3.02 & I & Yes & $0.43 \pm 0.06$ & $0.31 \pm 0.05$ & $3.45 \pm 0.09$ & $2.79 \pm 0.07$ & $1.47 \pm 0.08$ & $0.19 \pm 0.03$ & Composite \\
\hline F13479+3008 0.229 J135014.63+295318.1 & 12.18 & & & $\mathrm{n}$ & No & $3.29 \pm 0.05$ & $3.07 \pm 0.05$ & $13.62 \pm 0.12$ & $3.75 \pm 0.05$ & $3.33 \pm 0.07$ & $0.35 \pm 0.03$ & Starburst \\
\hline $\mathrm{F} 13485+37390.248 \mathrm{~J} 135043.17+372434.0$ & 12.27 & 2.7 & 1.84 & I & Yes & $0.40 \pm 0.06$ & $0.38 \pm 0.04$ & $4.20 \pm 0.10$ & $3.97 \pm 0.08$ & $1.41 \pm 0.11$ & $0.33 \pm 0.05$ & Composite \\
\hline $\mathrm{F} 13515+0317 \quad 0.278 \mathrm{~J} 135404.79+030242.6$ & 12.22 & & 2.53 & I & Yes & $0.43 \pm 0.03$ & $3.40 \pm 0.56$ & $4.63 \pm 0.09$ & $4.13 \pm 0.09$ & $1.70 \pm 0.08$ & $0.48 \pm 0.06$ & Seyfert \\
\hline 6-0108 0.199 J1 & 12.02 & & 0.81 & I & No & $0.40 \pm 0.04$ & $0.22 \pm 0.03$ & $3.12 \pm 0.05$ & $1.70 \pm 0.04$ & $1.12 \pm 0.05$ & $0.21 \pm 0.04$ & Composite \\
\hline F13539+2920 0.109 J135 & 12.11 & 11.6 & 10.70 & I & Yes & $1.29 \pm 0.05$ & $1.44 \pm 0.05$ & $14.26 \pm 0.11$ & $8.73 \pm 0.08$ & $6.84 \pm 0.08$ & $1.43 \pm 0.05$ & Composite \\
\hline $\mathrm{F} 13573+54290.253 \mathrm{~J} 135$ & 12.10 & & 2.09 & $\mathrm{n}$ & No & & & & & & & \\
\hline F14014+3718 0.211 J140337.76+370355.5 & 12.10 & 2.4 & 1.96 & III & Yes & $2.69 \pm 0.06$ & $2.70 \pm 0.04$ & $14.58 \pm 0.13$ & $5.70 \pm 0.08$ & $3.55 \pm 0.07$ & $0.40 \pm 0.05$ & Starburst \\
\hline F14026+4341 0.323 J140438.80+432707.4 & 12.71 & & 1.46 & $\mathrm{I}$ & Yes & & & & & & & \\
\hline $\mathrm{F} 14041+01170.236 \mathrm{~J} 140638.21+010254.6$ & 12.48 & 14.6 & 9.19 & $\mathrm{n}$ & Yes & $1.02 \pm 0.03$ & $2.73 \pm 0.12$ & $49.51 \pm 0.07$ & $1.68 \pm 0.10$ & $2.21 \pm 0.07$ & $0.99 \pm 0.07$ & Composite \\
\hline F14060+2919 0.117 J140819.03+290447.0 & 12.13 & 9.3 & 7.86 & I & Yes & $6.38 \pm 0.09$ & $5.03 \pm 0.09$ & $45.34 \pm 0.32$ & $20.29 \pm 0.17$ & $13.78 \pm 0.13$ & $1.82 \pm 0.06$ & Composite \\
\hline F14060-0304 0.353 J140837.68-031900.8 & 12.52 & & 2.14 & $\mathrm{n}$ & Yes & & & & & & & \\
\hline $\mathrm{F} 14082+02050.201 \mathrm{~J} 141049.55+015135.0$ & 12.07 & & 2.09 & I & $\mathrm{Ye}$ & & & & & & & \\
\hline F14088+0212 0.202 J141122.59+015815.1 & 12.05 & & 1.02 & I & Yes & & & & & & & \\
\hline
\end{tabular}


Table 1-Continued

\begin{tabular}{|c|c|c|c|c|c|c|c|c|c|c|c|c|c|c|}
\hline IRAS Name & $z_{\mathrm{sdss}}$ & SDSS Name & $\log \left(\frac{L_{I R}}{L_{\odot}}\right)$ & $\begin{array}{c}S_{1.4 \mathrm{GHS}}^{\mathrm{NVSS}} \\
(\mathrm{mJy})\end{array}$ & $\begin{array}{c}S_{1.4 \mathrm{GHz}}^{\mathrm{FIRST}} \\
(\mathrm{mJy})\end{array}$ & Note & NED & $f_{\mathrm{H}_{\beta}}$ & $f_{\mathrm{O}_{\mathrm{III}} 5007}$ & $f_{\mathrm{H}_{\alpha}}$ & $f_{\mathrm{N}_{\text {II }} 6583}$ & $f_{\mathrm{S}_{\mathrm{II}}}$ & $f_{\mathrm{O}_{\mathrm{I}} 6300}$ & Note \\
\hline (1) & $(2)$ & (3) & (4) & $(5)$ & (6) & (7) & $(8)$ & (9) & (10) & (11) & $(12)$ & (13) & (14) & $(15)$ \\
\hline
\end{tabular}

\begin{tabular}{|c|c|c|c|c|c|c|c|c|c|c|c|}
\hline $\mathrm{F} 14146+2353 \quad 0.282 \mathrm{~J} 141655.53+234018.2$ & 12.14 & & 1.28 & $\mathrm{n}$ & No & & & & & & \\
\hline F14166+6514 0.364 J141753.69+650025.2 & 12.71 & & & III & Yes & & & & & & \\
\hline $\mathrm{F} 14167+4247 \quad 0.421 \mathrm{~J} 141842.22+423343.3$ & 12.70 & & 1.25 & $\mathrm{n}$ & Yes & & & & & & \\
\hline $\mathrm{F} 14170+45450.150 \mathrm{~J} 141858.85+453212.7$ & 12.03 & 4.9 & 5.86 & I & Yes & & & & & & \\
\hline F14202+2615 0.159 J142231.37+260205.1 & 12.36 & 9.8 & 8.31 & II & Yes & $5.28 \pm 0.07$ & $4.72 \pm 0.07$ & $29.29 \pm 0.24$ & $13.13 \pm 0.13$ & $8.74 \pm 0.100 .98 \pm 0.05$ & Composite \\
\hline F14204+4533 0.167 J142221.85+452011.9 & 12.01 & 7.4 & 7.87 & $\mathrm{n}$ & Yes & $4.68 \pm 0.63$ & $4.77 \pm 0.26$ & $28.24 \pm 0.22$ & $12.61 \pm 0.12$ & $8.21 \pm 0.12 \quad 1.20 \pm 0.06$ & Composite \\
\hline F14248-0045 0.162 J142727.28-005841.1 & 12.18 & 5.4 & 3.51 & III & Yes & $0.77 \pm 0.04$ & $0.60 \pm 0.05$ & $9.73 \pm 0.11$ & $5.56 \pm 0.08$ & $3.31 \pm 0.080 .58 \pm 0.06$ & Composite \\
\hline F14298+5259 0.363 J143130.12+524532.8 & 12.43 & & & $\mathrm{n}$ & Yes & & & & & & \\
\hline $\mathrm{F} 14302+12430.332 \mathrm{~J} 143245.00+122951.3$ & 12.46 & & & $\mathrm{n}$ & No & & & & & & \\
\hline $\mathrm{F} 14312+2825 \quad 0.174$ J143327.52+281159.8 & 12.22 & 6.0 & 5.63 & I & Yes & $0.60 \pm 0.08$ & $5.07 \pm 0.09$ & $8.20 \pm 0.09$ & $7.47 \pm 0.08$ & $2.68 \pm 0.07 \quad 0.53 \pm 0.04$ & Seyfert \\
\hline $\mathrm{F} 14315+29550.527 \mathrm{~J} 143344.53+294248.0$ & 12.82 & & & $\mathrm{n}$ & No & & & & & & \\
\hline F14318-0252 0.187 J143425.31-030614.4 & 12.10 & & & $\mathrm{n}$ & No & $3.68 \pm 0.06$ & $9.41 \pm 0.07$ & $14.21 \pm 0.10$ & $2.44 \pm 0.05$ & $3.89 \pm 0.070 .55 \pm 0.04$ & Starburst \\
\hline F14330+0141 0.232 J143535.54+012834.8 & 12.16 & 14.6 & 15.08 & I & Yes & $0.16 \pm 0.03$ & $1.54 \pm 0.05$ & $1.82 \pm 0.05$ & $1.83 \pm 0.05$ & $0.93 \pm 0.09$ & Seyfert \\
\hline F14336-0147 0.150 J143610.59-020055.6 & 12.02 & & & I & No & $1.04 \pm 0.15$ & $1.61 \pm 0.33$ & $4.17 \pm 0.06$ & $1.24 \pm 0.05$ & $0.44 \pm 0.04$ & Starburst \\
\hline F14351+3553 0.362 J143715.26+354006.7 & 12.42 & & & $\mathrm{n}$ & Yes & & & & & & \\
\hline F14374+0120 0.456 J144001.25+010743.2 & 12.80 & & & $\mathrm{n}$ & Yes & & & & & & \\
\hline F14379+5420 0.268 J143930.59+540807.4 & 12.22 & 3.6 & 1.93 & I & Yes & $0.91 \pm 0.04$ & $0.98 \pm 0.03$ & $5.66 \pm 0.07$ & $2.07 \pm 0.09$ & $1.82 \pm 0.06 \quad 0.32 \pm 0.05$ & Starburst \\
\hline F14390+6209 0.275 J144012.76+615633.2 & 12.31 & 3.4 & 2.88 & & Yes & & & & & & \\
\hline F14394+5332 0.105 J144104.38+532008.7 & 12.08 & 42.2 & 39.55 & II & Yes & & & & & & \\
\hline $\mathrm{F} 14402+01080.243 \mathrm{~J} 144247.57+005532.0$ & 12.08 & & & $\mathrm{n}$ & No & & & & & & \\
\hline F14413+3730 0.259 J144319.55+371800.9 & 12.20 & & 1.76 & I & Yes & & & & & & \\
\hline F14483+0059 0.334 J145054.38+004646.8 & 12.37 & & 1.29 & I & No & & & & & & \\
\hline F14488+3521 0.206 J145054.16+350837.8 & 12.30 & 6.2 & 5.26 & $\mathrm{n}$ & Yes & $3.65 \pm 0.09$ & $5.88 \pm 0.09$ & $28.86 \pm 0.25$ & $19.19 \pm 0.19$ & $7.45 \pm 0.13 \quad 1.23 \pm 0.11$ & Composite \\
\hline F14513-0235 0.209 J145355.90-024745.4 & 12.10 & & 1.17 & II & Yes & $1.06 \pm 0.17$ & $0.54 \pm 0.04$ & $5.25 \pm 0.07$ & $2.28 \pm 0.06$ & $1.91 \pm 0.06 \quad 0.26 \pm 0.04$ & Starburst \\
\hline $\mathrm{F} 14541+38130.283 \mathrm{~J} 145608.63+380038.6$ & 12.01 & & & $\mathrm{n}$ & No & & & & & & \\
\hline F14541+4906 0.247 J145549.42+485436.3 & 12.31 & 7.0 & 6.35 & I & Yes & $4.39 \pm 0.18$ & $35.01 \pm 0.39$ & $14.91 \pm 0.14$ & $7.92 \pm 0.09$ & $3.80 \pm 0.090 .95 \pm 0.06$ & Seyfert \\
\hline F14541+5734 0.301 J145531.90+572258.1 & 12.14 & & & $\mathrm{n}$ & No & $2.83 \pm 0.05$ & $5.28 \pm 0.06$ & $11.68 \pm 0.12$ & $3.32 \pm 0.07$ & $2.56 \pm 0.100 .46 \pm 0.05$ & Starburst \\
\hline F14560+0845 0.307 J145829.97+083400.3 & 12.36 & & 2.28 & I & No & & & & & & \\
\hline F15002+4945 0.337 J150150.52+493338.4 & 12.52 & 3.1 & 3.09 & $\mathrm{n}$ & Yes & $1.16 \pm 0.04$ & $1.46 \pm 0.04$ & $6.78 \pm 0.15$ & $3.76 \pm 0.10$ & $1.98 \pm 0.130 .48 \pm 0.08$ & Composite \\
\hline $\mathrm{F} 15004+0351 \quad 0.218 \mathrm{~J} 150259.01+034003.9$ & 12.13 & & 2.10 & $\mathrm{n}$ & Yes & $1.22 \pm 0.05$ & $0.47 \pm 0.03$ & $8.26 \pm 0.11$ & $5.47 \pm 0.08$ & $3.01 \pm 0.08 \quad 0.39 \pm 0.04$ & Composite \\
\hline F15023+5404 0.280 J150351.02+535243.7 & 12.11 & & & I & No & $0.61 \pm 0.06$ & $0.18 \pm 0.03$ & $3.58 \pm 0.10$ & $1.46 \pm 0.09$ & $1.05 \pm 0.090 .00 \pm 0.00$ & Starburst \\
\hline
\end{tabular}


Table 1-Continued

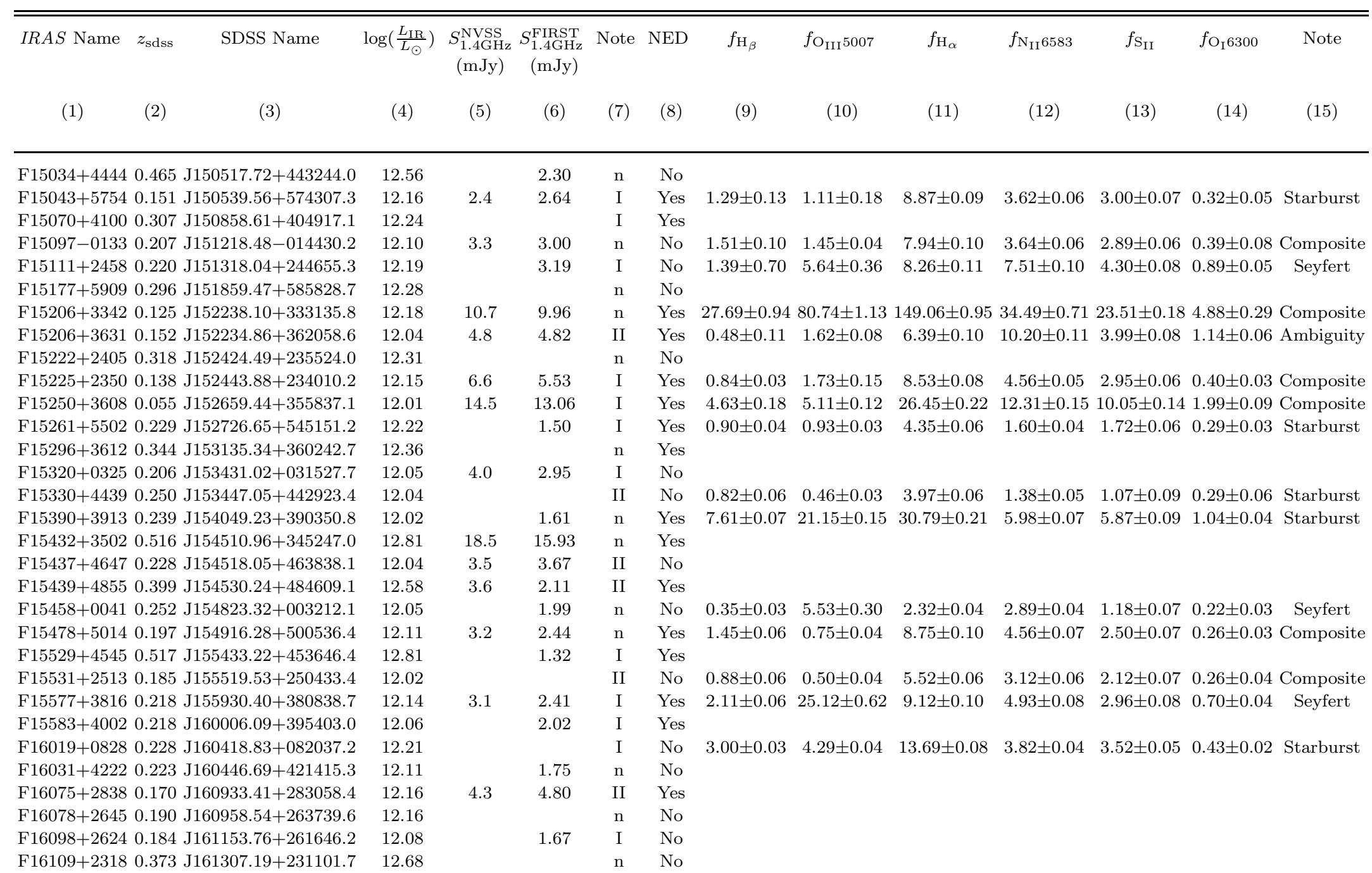


Table 1-Continued

\begin{tabular}{|c|c|c|c|c|c|c|c|c|c|c|c|c|c|c|}
\hline IRAS Name & (2) & SDSS Name & $\begin{array}{c}\log \left(\frac{L_{\mathrm{IR}}}{L_{\odot}}\right) \\
(4)\end{array}$ & $\begin{array}{c}S_{1.4 \mathrm{GHz}}^{\mathrm{NVSS}} \\
(\mathrm{mJy}) \\
(5)\end{array}$ & $\begin{array}{c}\begin{array}{c}S_{1.4 \mathrm{GHz}}^{\mathrm{FIRST}} \\
(\mathrm{mJy})\end{array} \\
(6)\end{array}$ & Note & NED & $f_{\mathrm{H}_{\beta}}$ & $\begin{array}{c}f_{\mathrm{O}_{\mathrm{III}} 5007} \\
(10)\end{array}$ & $f_{\mathrm{H}_{\alpha}}$ & $\begin{array}{c}f_{\mathrm{N}_{\mathrm{II}} 6583} \\
(12)\end{array}$ & $\begin{array}{l}f_{\mathrm{S}_{\mathrm{II}}} \\
(13)\end{array}$ & $\begin{array}{r}f_{\mathrm{O}_{\mathrm{I}} 6300} \\
(14)\end{array}$ & Note \\
\hline $\mathrm{F} 16116+0638$ & 0.241 & $1 \mathrm{~J} 161407.70+063114.1$ & 12.14 & & 1.32 & I & No & & & & & & & \\
\hline $\mathrm{F} 16117+4904$ & 0.241 & $1 \mathrm{~J} 161315.95+485721.9$ & 12.04 & 4.8 & 4.32 & $\mathrm{n}$ & No & & & & & & & \\
\hline $\mathrm{F} 16122+1531$ & 0.308 & $8 \mathrm{~J} 161431.72+152421.9$ & 12.42 & 24.2 & 20.27 & $\mathrm{n}$ & No & & & & & & & \\
\hline $\mathrm{F} 16126+1953$ & 0.252 & 2 J161448.24+194609.8 & 12.27 & & 2.93 & I & No & & & & & & & \\
\hline $\mathrm{F} 16133+2107$ & 0.091 & $1 \mathrm{~J} 161534.13+210019.7$ & 12.00 & 9.4 & 11.24 & I & Yes & $0.61 \pm 0.08$ & $0.99 \pm 0.06$ & $6.63 \pm 0.08$ & $6.20 \pm 0.08$ & $4.85 \pm 0.09$ & $1.14 \pm 0.06$ & LINERs \\
\hline $\mathrm{F} 16148+2104$ & 0.415 & 5 J161657.59+205746.6 & 12.85 & & & II & No & & & & & & & \\
\hline $\mathrm{F} 16172+4432$ & 0.335 & $5 \mathrm{~J} 161849.25+442517.3$ & 12.41 & 3.0 & 3.09 & I & No & & & & & & & \\
\hline $\mathrm{F} 16264+3157$ & 0.361 & $1 \mathrm{~J} 162824.02+315028.3$ & 12.37 & & 1.51 & $\mathrm{n}$ & Yes & $0.06 \pm 0.02$ & $1.57 \pm 0.03$ & $1.15 \pm 0.14$ & $1.30 \pm 0.13$ & $1.02 \pm 0.12$ & $0.14 \pm 0.04$ & Seyfert \\
\hline $\mathrm{F} 16300+1558$ & 0.242 & $2 \mathrm{~J} 163221.37+155145.5$ & 12.77 & 7.5 & 5.93 & I & Yes & $0.25 \pm 0.03$ & $0.73 \pm 0.04$ & $3.85 \pm 0.07$ & $2.25 \pm 0.06$ & $1.97 \pm 0.10$ & $0.44 \pm 0.05$ & Seyfert \\
\hline $\mathrm{F} 16301+4617$ & 0.329 & $9 \mathrm{~J} 163134.12+461050.6$ & 12.29 & & 1.04 & I & Yes & $1.10 \pm 0.06$ & $0.84 \pm 0.03$ & $5.53 \pm 0.09$ & $2.44 \pm 0.08$ & $1.51 \pm 0.10$ & $0.15 \pm 0.04$ & Composite \\
\hline $\mathrm{F} 16403+2537$ & 0.160 & J J164223.46+253147.6 & 12.03 & & 4.40 & $\mathrm{n}$ & No & $3.36 \pm 0.08$ & $2.05 \pm 0.08$ & $25.16 \pm 0.25$ & $14.10 \pm 0.16$ & $8.72 \pm 0.14$ & $1.09 \pm 0.08$ & Composite \\
\hline $\mathrm{F} 16413+3954$ & 0.594 & 4 J164258.81+394837.0 & 13.39 & 7098.6 & 6598.61 & $\mathrm{n}$ & Yes & & & & & & & \\
\hline $\mathrm{F} 16474+3430$ & 0.113 & 3 J164914.09+342513.2 & 12.22 & 11.1 & & II & Yes & $1.54 \pm 0.05$ & $1.00 \pm 0.05$ & $7.71 \pm 0.09$ & $3.14 \pm 0.05$ & $2.52 \pm 0.06$ & $0.26 \pm 0.03$ & Starburst \\
\hline $\mathrm{F} 16533+6216$ & 0.106 & $6 \mathrm{~J} 165352.11+621149.7$ & 12.02 & 16.5 & 14.43 & $\mathrm{n}$ & Yes & $5.90 \pm 0.09$ & $3.13 \pm 0.07$ & $36.78 \pm 0.24$ & $20.22 \pm 0.16$ & $12.02 \pm 0.11$ & $1.67 \pm 0.06$ & Composite \\
\hline $\mathrm{F} 17051+3824$ & 0.168 & $8 \mathrm{~J} 170653.27+382007.0$ & 12.23 & 4.4 & 4.30 & I & Yes & $0.24 \pm 0.07$ & $0.71 \pm 0.07$ & $2.23 \pm 0.06$ & $3.22 \pm 0.06$ & $2.25 \pm 0.09$ & $0.50 \pm 0.06$ & LINERs \\
\hline $\mathrm{F} 17081+3300$ & 0.279 & 9 J171000.75+325658.0 & 12.20 & & & $\mathrm{n}$ & Yes & $0.87 \pm 0.03$ & $3.29 \pm 0.05$ & $6.42 \pm 0.08$ & $4.89 \pm 0.07$ & $1.74 \pm 0.07$ & $0.39 \pm 0.04$ & Seyfert \\
\hline $\mathrm{F} 17175+6603$ & 0.292 & 2 J171737.96+655939.3 & 12.25 & & & $\mathrm{n}$ & Yes & & & & & & & \\
\hline $\mathrm{F} 17214+2845$ & 0.241 & 1 J172322.64+284249.6 & 12.32 & & & $\mathrm{n}$ & No & $0.91 \pm 0.03$ & $0.43 \pm 0.02$ & $4.99 \pm 0.06$ & $1.80 \pm 0.04$ & $1.54 \pm 0.06$ & $0.21 \pm 0.04$ & Starburst \\
\hline $\mathrm{F} 17234+6228$ & 0.240 & 0 J172351.74+622519.6 & 12.14 & 5.7 & 1.93 & $\mathrm{n}$ & Yes & & & & & & & \\
\hline F20522-0120 & 0.173 & 3 J205450.55-010831.3 & 12.22 & & & I & No & $4.41 \pm 0.06$ & $6.20 \pm 0.07$ & $17.96 \pm 0.12$ & $5.71 \pm 0.06$ & $4.68 \pm 0.07$ & $0.60 \pm 0.03$ & Starburst \\
\hline $\mathrm{F} 21045+1058$ & 0.166 & 6 J210654.94+111007.8 & 12.09 & & & I & No & $0.14 \pm 0.03$ & $0.35 \pm 0.04$ & $2.52 \pm 0.05$ & $1.97 \pm 0.04$ & $1.34 \pm 0.07$ & $0.55 \pm 0.06$ & Ambiguity \\
\hline F21234-0023 & 0.458 & 8 J212559.03-001044.4 & 13.08 & & & $\mathrm{n}$ & Yes & & & & & & & \\
\hline $\mathrm{F} 21461+1117$ & 0.151 & $1 \mathrm{~J} 214833.44+113147.8$ & 12.02 & 10.6 & & I & Yes & & & & & & & \\
\hline $\mathrm{F} 22015+0045$ & 0.289 & 9 J220405.30+005917.5 & 12.31 & & & $\mathrm{n}$ & Yes & $3.92 \pm 0.05$ & $7.14 \pm 0.07$ & $17.32 \pm 0.17$ & $5.07 \pm 0.09$ & $4.63 \pm 0.13$ & $1.07 \pm 0.05$ & Ambiguity \\
\hline F22098-0748 & 0.143 & 3 J221232.09-073334.0 & 12.00 & 4.8 & 4.63 & I & Yes & $1.67 \pm 0.06$ & $0.89 \pm 0.06$ & $12.68 \pm 0.11$ & $7.21 \pm 0.08$ & $3.99 \pm 0.08$ & $0.48 \pm 0.05$ & Composite \\
\hline F22239-0916 & 0.298 & $8 \mathrm{~J} 222634.05-090106.1$ & 12.39 & & 1.72 & $\mathrm{n}$ & Yes & $0.20 \pm 0.03$ & $0.60 \pm 0.05$ & $2.78 \pm 0.07$ & $2.24 \pm 0.07$ & $0.36 \pm 0.07$ & $0.10 \pm 0.04$ & Ambiguity \\
\hline $\mathrm{F} 22532+1233$ & 0.356 & $6 \mathrm{~J} 225545.29+124943.4$ & 12.70 & & & I & Yes & & & & & & & \\
\hline F23051-0100 & 0.362 & 2 J230743.26-004404.0 & 12.55 & & & $\mathrm{n}$ & No & & & & & & & \\
\hline $\mathrm{F} 23254+1429$ & 0.156 & $6 \mathrm{~J} 232800.79+144646.7$ & 12.02 & & & $\mathrm{n}$ & No & & & & & & & \\
\hline
\end{tabular}


Note. — Column 1: IRAS name; Column 2: redshift from SDSS; Column 3: SDSS name of the object; Column 4: infrared luminosity; Column 5 and 6: radio fluxes from NVSS and FIRST, respectively; Column 7: classes (I, II, III) for interaction features, and "n" stands for not clear; Column 8: NED identifications. "Yes": the redshift and/or the counterpart provided in the NED is consistent with ours; "No": the redshift is not listed in the NED; Column 9 to 14: fluxes of $\mathrm{H}_{\beta}$, $\left[\mathrm{O}_{\mathrm{III}}\right] 5007, \mathrm{H}_{\alpha},\left[\mathrm{N}_{\mathrm{II}}\right] 6583,\left[\mathrm{~S}_{\mathrm{II}}\right] 6716+6731,\left[\mathrm{O}_{\mathrm{I}}\right] 6300$, all in units of $10^{-15} \mathrm{ergs}^{-1} \mathrm{~cm}^{-2}$; Column 15: note for the type of the galaxy according to the BPT diagram. 
Table 2. The parameters of the Type I ULIRG sample.

\begin{tabular}{|c|c|c|c|c|c|c|c|c|c|c|}
\hline IRAS Name & $(2)$ & $f_{(5100 \AA)}$ & $\begin{array}{c}\text { FWHM } \\
\mathrm{H}_{\beta} \\
\left(\mathrm{km} \mathrm{s}^{-1}\right) \\
(4)\end{array}$ & $\begin{array}{c}\text { FWHM } \\
{\left[\mathrm{O}_{\mathrm{III}}\right]} \\
\left(\mathrm{km} \mathrm{s}^{-1}\right) \\
(5)\end{array}$ & $\begin{array}{c}\text { FWHM } \\
{\left[\mathrm{O}_{\text {III }}\right] \text { NL core }} \\
\left(\mathrm{km} \mathrm{s}^{-1}\right) \\
(6)\end{array}$ & $\begin{array}{c}\text { FWHM } \\
{\left[\mathrm{S}_{\mathrm{II}}\right]} \\
\left(\mathrm{km} \mathrm{s}^{-1}\right) \\
(7)\end{array}$ & $\begin{array}{c}M_{\mathrm{BH}} \\
10^{7} M_{\odot} \\
(8)\end{array}$ & $\frac{L_{\mathrm{bol}}}{L_{\mathrm{Edd}}}$ & $\log \left(\frac{L_{\mathrm{IR}}}{L_{\odot}}\right)$ & quality \\
\hline F01572+0009 & 0.163 & $134.88_{-22.09}^{+25.23}$ & 3141 & 689 & $504_{-8}^{+7}$ & $592_{-26}^{+26}$ & $10.21 \pm 2.65$ & 0.35 & 12.52 & 1 \\
\hline F02486-0714 & 0.327 & $17.42_{-6.92}^{+9.87}$ & 1799 & 748 & $564_{-14}^{+13}$ & $430_{-28}^{+28}$ & $2.51 \pm 1.01$ & 0.89 & 12.32 & 1 \\
\hline F07548+4227 & 0.211 & $59.55_{-10.81}^{+12.54}$ & 3691 & 449 & $357_{-5}^{+6}$ & $412_{-12}^{+12}$ & $12.15 \pm 3.06$ & 0.23 & 12.13 & 1 \\
\hline F07578+5148 & 0.196 & $22.71_{-7.81}^{+10.51}$ & 3201 & 461 & $385_{-12}^{+11}$ & $405_{-32}^{+32}$ & $4.59 \pm 1.53$ & 0.20 & 12.07 & 1 \\
\hline F08209+2458 & 0.234 & $10.35_{-6.00}^{+10.63}$ & 949 & 886 & 886 & & $0.32 \pm 0.24$ & 1.93 & 12.19 & 2 \\
\hline F08238+0752 & 0.311 & $66.30_{-13.47}^{+15.92}$ & 3547 & 724 & $669_{-30}^{+31}$ & & $20.53 \pm 6.66$ & 0.37 & 12.41 & 1 \\
\hline $\mathrm{F} 08407+2630$ & 0.257 & $31.67_{-8.18}^{+10.16}$ & 3156 & 509 & $462_{-8}^{+9}$ & $369_{-50}^{+50}$ & $7.93 \pm 2.25$ & 0.29 & 12.10 & 1 \\
\hline F08435+3141 & 0.386 & $7.72_{-5.00}^{+9.180}$ & 2110 & 2185 & $2185^{-8}$ & & $2.66 \pm 2.14$ & 0.55 & 12.46 & 2 \\
\hline F08542+1920 & 0.331 & $41.29_{-9.85}^{+11.99}$ & 4362 & 2084 & 2084 & & $25.40 \pm 8.08$ & 0.21 & 12.46 & 2 \\
\hline $\mathrm{F} 09005+2253$ & 0.290 & $\begin{array}{l}15.97_{-5.99}^{+8.34} \\
\end{array}$ & 7577 & 413 & $413_{-9}^{+9}$ & $390_{-25}^{+25}$ & $35.62 \pm 13.16$ & 0.04 & 12.24 & 1 \\
\hline F09008+3643 & 0.288 & $6.04_{-4.98}^{+13.30}$ & 2552 & 1485 & 1485 & & $2.21 \pm 3.01$ & 0.26 & 12.55 & 2 \\
\hline F09105+4108 & 0.442 & $12.52_{-5.37}^{+7.98}$ & 1362 & 659 & $492_{-3}^{+3}$ & & $1.81 \pm 0.81$ & 1.80 & 12.87 & 1 \\
\hline F09220+2759 & 0.531 & $5.86_{-4.14}^{+8.91}$ & 2335 & 970 & 641 & & $4.38 \pm 4.17$ & 0.54 & 12.92 & 2 \\
\hline F09438+4735 & 0.539 & $21.67_{-9.99}^{+15.28}$ & 4804 & 784 & 784 & & $42.08 \pm 22.50$ & 0.22 & 13.01 & 2 \\
\hline F09591+2045 & 0.357 & $13.42_{-6.16}^{+9.39}$ & 5479 & 701 & 265 & & $22.48 \pm 11.07$ & 0.09 & 12.50 & 2 \\
\hline F10015-0018 & 0.289 & $13.09_{-5.86}^{+8.81}$ & 1562 & 736 & 265 & $239_{-24}^{+24}$ & $1.34 \pm 0.61$ & 0.94 & 12.52 & 2 \\
\hline $\mathrm{F} 10026+4347$ & 0.179 & $80.56_{-18.73}^{+22.74}$ & 2627 & 1772 & 1772 & & $5.92 \pm 1.62$ & 0.45 & 12.08 & 2 \\
\hline $\mathrm{F} 10106+2227$ & 0.274 & $27.74_{-7.73}^{+9.80}$ & 3752 & 1048 & $1048_{-70}^{+69}$ & $277_{-33}^{+32}$ & $11.30 \pm 3.39$ & 0.21 & 12.15 & 1 \\
\hline F10234+3052 & 0.340 & $29.43_{-9.63}^{+12.75}$ & 2980 & 665 & $517_{-19}^{+19}$ & $448_{-87}^{+87}$ & $10.02 \pm 3.60$ & 0.41 & 12.47 & 1 \\
\hline $\mathrm{F} 10372+4801$ & 0.486 & $9.39_{-5.37}^{+9.44}$ & 1109 & 641 & $559_{-5}^{+6}$ & & $1.16 \pm 0.84$ & 2.65 & 12.81 & 1 \\
\hline F10531+5531 & 0.256 & $17.81_{-7.77}^{+11.56}$ & 3671 & 557 & $557_{-35}^{+35}$ & $304_{-56}^{+55}$ & $7.51 \pm 3.27$ & 0.17 & 12.01 & 1 \\
\hline F10538+3309 & 0.457 & $5.43_{-4.08}^{+9.49}$ & 2841 & 461 & 461 & & $4.97 \pm 5.44$ & 0.31 & 12.62 & 2 \\
\hline $\mathrm{F} 11028+3442$ & 0.509 & $99.27_{-20.19}^{+23.88}$ & 4347 & 2311 & 2311 & & $80.14 \pm 36.16$ & 0.45 & 12.86 & 2 \\
\hline F11066+4242 & 0.232 & $16.16_{-7.37}^{+11.20}$ & 6845 & 527 & $527_{-19}^{+19}$ & & $21.47 \pm 9.91$ & 0.04 & 12.11 & 1 \\
\hline $\mathrm{F} 11070+4249$ & 0.261 & $14.34_{-6.73}^{+10.39}$ & 1230 & 904 & $725_{-14}^{+13}$ & $534_{-18}^{+17}$ & $0.76 \pm 0.36$ & 1.44 & 12.34 & 1 \\
\hline $\mathrm{F} 11134+0225$ & 0.211 & $61.01_{-13.28}^{+15.93}$ & 3398 & 790 & $467_{-32}^{+143}$ & & $10.45 \pm 2.82$ & 0.28 & 12.01 & 1 \\
\hline $\mathrm{F} 11162+6020$ & 0.264 & $18.40_{-8.35}^{+12.65}$ & 1535 & 1156 & 779 & & $1.40 \pm 0.64$ & 1.03 & 12.55 & 2 \\
\hline $\mathrm{F} 11163+3207$ & 0.261 & $15.03_{-7.34}^{+11.59}$ & 1369 & 814 & $570_{-16}^{+16}$ & & $0.97 \pm 0.65$ & 1.18 & 12.22 & 1 \\
\hline F11206+3639 & 0.242 & $\begin{array}{l}11.77_{-5.90}^{+9.45} \\
\end{array}$ & 990 & 641 & $506_{-9}^{+9}$ & & $0.39 \pm 0.21$ & 1.94 & 12.42 & 1 \\
\hline $\mathrm{F} 11307+0449$ & 0.248 & $19.10_{-7.31}^{+10.23}$ & 2593 & 335 & $335_{-15}^{+15}$ & $261_{-83}^{+83}$ & $3.74 \pm 1.40$ & 0.35 & 12.12 & 1 \\
\hline $\mathrm{F} 11394+0108$ & 0.245 & $14.81_{-6.07}^{+8.76}$ & 1142 & 359 & $328_{-8}^{+9}$ & $427_{-10}^{+9}$ & $0.61 \pm 0.27$ & 1.61 & 12.22 & 1 \\
\hline $\mathrm{F} 11417+1151$ & 0.271 & $100.11_{-20.80}^{+24.62}$ & 6058 & 581 & $440_{-15}^{+16}$ & & $63.47 \pm 21.14$ & 0.13 & 12.33 & 1 \\
\hline F11553-0259 & 0.215 & $25.82_{-8.98}^{+12.13}$ & 2534 & 1180 & $961_{-17}^{+17}$ & & $3.53 \pm 1.19$ & 0.36 & 12.06 & 1 \\
\hline $\mathrm{F} 11557+1342$ & 0.439 & $30.18_{-8.95}^{+11.52}$ & 3629 & 311 & $268_{-8}^{+8}$ & & $21.78 \pm 8.13$ & 0.36 & 12.66 & 1 \\
\hline $\mathrm{F} 12043+5215$ & 0.398 & $8.26_{-4.84}^{+8.80}$ & 2012 & 1401 & 1401 & & $2.63 \pm 1.80$ & 0.64 & 12.51 & 2 \\
\hline F12136+3919 & 0.334 & $8.90_{-5.1}^{+9}$ & 4036 & 455 & $455_{-31}^{+30}$ & & $8.64 \pm 5.68$ & 0.14 & 12.70 & 1 \\
\hline $\mathrm{F} 12433+6540$ & 0.320 & $33.34_{-9.39}^{+11.95}$ & 3097 & 365 & $280_{-19}^{+18}$ & $197_{-28}^{+28}$ & $10.71 \pm 3.50$ & 0.38 & 12.36 & 1 \\
\hline F12489+6619 & 0.282 & $62.99_{-13.68}^{+16.40}$ & 3074 & 299 & $299_{-12}^{+19}$ & $371_{-39}^{+39}$ & $13.02 \pm 4.06$ & 0.44 & 12.17 & 1 \\
\hline $\mathrm{F} 13210+3932$ & 0.566 & $12.08_{-5.77}^{+9.09}$ & 3042 & 695 & 695 & & $12.69 \pm 6.94$ & 0.45 & 12.93 & 2 \\
\hline F13342+3932 & 0.179 & $58.35_{-14.24}^{+17.40}$ & 5789 & 503 & $467_{-7}^{+6}$ & $435_{-13}^{+13}$ & $23.59 \pm 6.38$ & 0.08 & 12.37 & 1 \\
\hline F13403-0038 & 0.326 & $48.91_{-9.69}^{+11.42}$ & 4456 & 1209 & $865_{-59}^{+60}$ & & $28.76 \pm 8.88$ & 0.22 & 12.39 & 1 \\
\hline $\mathrm{F} 13451+1232 \mathrm{w}$ & 0.121 & $22.59_{-7.95}^{+10.78}$ & 2249 & 1383 & 1087 & & $1.18 \pm 0.42$ & 0.27 & 12.19 & 2 \\
\hline
\end{tabular}


Table 2-Continued

\begin{tabular}{|c|c|c|c|c|c|c|c|c|c|c|}
\hline$I R A S$ Name & $(2)$ & $f_{(5100 \AA)}$ & $\begin{array}{c}\text { FWHM } \\
\mathrm{H}_{\beta} \\
\left(\mathrm{km} \mathrm{s}^{-1}\right) \\
(4)\end{array}$ & $\begin{array}{c}\text { FWHM } \\
{\left[\mathrm{O}_{\mathrm{III}}\right]} \\
\left(\mathrm{km} \mathrm{s}^{-1}\right) \\
(5)\end{array}$ & $\begin{array}{c}\text { FWHM } \\
{\left[\mathrm{O}_{\mathrm{III}}\right] \mathrm{NL} \text { core }} \\
\left(\mathrm{km} \mathrm{s}^{-1}\right) \\
(6)\end{array}$ & $\begin{array}{c}\text { FWHM } \\
{\left[\mathrm{S}_{\mathrm{II}}\right]} \\
\left(\mathrm{km} \mathrm{s}^{-1}\right) \\
(7)\end{array}$ & $\begin{array}{c}M_{\mathrm{BH}} \\
10^{7} M_{\odot} \\
(8)\end{array}$ & $\frac{L_{\mathrm{bol}}}{L_{\mathrm{Edd}}}$ & $\log \left(\frac{L_{\mathrm{IR}}}{L_{\odot}}\right)$ & quality \\
\hline F14026+4341 & 0.323 & $225.42_{-37.00}^{+42.23}$ & 5490 & 2084 & 2084 & & $109.45 \pm 45.99$ & 0.26 & 12.71 & 2 \\
\hline $\mathrm{F} 14082+0205$ & 0.201 & $14.00_{-6.41}^{+9.76}$ & 2161 & 742 & $742_{-24}^{+24}$ & $192_{-11}^{+11}$ & $1.61 \pm 0.78$ & 0.37 & 12.07 & 1 \\
\hline $\mathrm{F} 14166+6514$ & 0.364 & $11.46_{-5.97}^{+9.81}$ & 3775 & 533 & $533_{-29}^{+28}$ & & $9.97 \pm 5.55$ & 0.19 & 12.71 & 1 \\
\hline $\mathrm{F} 14167+4247$ & 0.421 & $61.33_{-14.74}^{+18.06}$ & 5346 & 515 & $412_{-20}^{+20}$ & & $68.55 \pm 26.57$ & 0.21 & 12.70 & 1 \\
\hline $\mathrm{F} 14302+1243$ & 0.332 & $9.75_{-5.49}^{+9.52}$ & 4677 & 365 & $346_{-10}^{+90}$ & & $12.16 \pm 7.66$ & 0.11 & 12.46 & 1 \\
\hline $\mathrm{F} 14315+2955$ & 0.527 & $28.53_{-9.25}^{+12.28}$ & 5973 & 581 & $412_{-24}^{+24}$ & & $74.42 \pm 31.04$ & 0.15 & 12.82 & 1 \\
\hline F14390+6209 & 0.275 & $54.52_{-10.95}^{+12.92}$ & 3375 & 479 & $346_{-8}^{+8}$ & $429_{-16}^{+17}$ & $13.88 \pm 4.03$ & 0.34 & 12.31 & 1 \\
\hline F14394+5332 & 0.105 & $43.82_{-12.31}^{+15.58}$ & 1827 & 1826 & 1828 & & $0.97 \pm 0.27$ & 0.46 & 12.08 & 2 \\
\hline $\mathrm{F} 14402+0108$ & 0.243 & $9.56_{-4.90}^{+7.95}$ & 4097 & 323 & $323_{-11}^{+10}$ & & $5.96 \pm 3.21$ & 0.10 & 12.08 & 1 \\
\hline $\mathrm{F} 14541+3813$ & 0.283 & $28.30_{-9.30}^{+12.38}$ & 7045 & 569 & $492_{-13}^{+11}$ & $551_{-109}^{+109}$ & $42.20 \pm 14.44$ & 0.06 & 12.01 & 1 \\
\hline $\mathrm{F} 15320+0325$ & 0.206 & $22.95_{-7.72}^{+10.32}$ & 904 & 581 & $254_{-12}^{+13}$ & & $0.39 \pm 0.14$ & 2.63 & 12.05 & 1 \\
\hline $\mathrm{F} 15432+3502$ & 0.516 & $51.61_{-13.05}^{+16.14}$ & 3817 & 1449 & $478_{-67}^{+67}$ & & $42.30 \pm 17.69$ & 0.46 & 12.81 & 1 \\
\hline $\mathrm{F} 15437+4647$ & 0.228 & $35.04_{-10.78}^{+14.00}$ & 4972 & 814 & $465_{-35}^{+35}$ & $491_{-16}^{+16}$ & $17.74 \pm 6.01$ & 0.11 & 12.04 & 1 \\
\hline $\mathrm{F} 15439+4855$ & 0.399 & $82.80_{-17.28}^{+20.52}$ & 5628 & 2090 & 2090 & & $84.43 \pm 32.91$ & 0.20 & 12.58 & 2 \\
\hline $\mathrm{F} 15529+4545$ & 0.517 & $8.92_{-5.93}^{+11.94}$ & 2931 & 772 & $587_{-31}^{+32}$ & & $8.57 \pm 7.32$ & 0.39 & 12.81 & 1 \\
\hline $\mathrm{F} 16122+1531$ & 0.308 & $13.71_{-5.75}^{+8.43}$ & 2041 & 2138 & 487 & $538_{-20}^{+21}$ & $2.56 \pm 1.12$ & 0.60 & 12.42 & 2 \\
\hline $\mathrm{F} 16172+4432$ & 0.335 & $57.28_{-12.83}^{+15.41}$ & 5150 & 497 & $391_{-10}^{+11}$ & & $43.97 \pm 14.72$ & 0.18 & 12.41 & 1 \\
\hline F16413+3954 & 0.594 & $271.96_{-34.84}^{+38.50}$ & 4439 & 1126 & 1126 & & $194.01 \pm 110.28$ & 0.74 & 13.39 & 2 \\
\hline $\mathrm{F} 17175+6603$ & 0.292 & $31.56_{-8.42}^{+10.58}$ & 5701 & 641 & $403_{-24}^{+24}$ & $511_{-70}^{+69}$ & $30.86 \pm 9.34$ & 0.10 & 12.25 & 1 \\
\hline $\mathrm{F} 17234+6228$ & 0.240 & $7.51_{-5.51}^{+12.34}$ & 1113 & 425 & $385_{-9}^{+9}$ & $728_{-36}^{+36}$ & $0.37 \pm 0.65$ & 1.28 & 12.14 & 1 \\
\hline
\end{tabular}

Note. - Column 1: IRAS name; Column 2: redshift; Column 3: continuum flux at $5100 \AA$ corrected by the use of $F_{\lambda}(5100 \AA)_{\text {rest }}=(1+z) F_{\lambda}((1+z) 5100 \AA)_{\text {obs }}$, where $z$ is the redshift , and the unit is $10^{-17} \mathrm{ergs}^{-1} \mathrm{~cm}^{-2} \AA^{-1}$; Column 4 to 7 : FWHM of the broad component of $\mathrm{H}_{\beta}$, the FWHM of the $\left[\mathrm{O}_{\mathrm{III}}\right] 5007$ profile, and the $\left[\mathrm{O}_{\mathrm{III}}\right] 5007 \mathrm{NL}$ core, as well as the $\left[\mathrm{S}_{\mathrm{II}}\right] 6716$, if available; Column 8: derived BH mass; Column 9: Eddington ratio; Column 10: infrared luminosity; Column 11: the quality of $\left[\mathrm{O}_{\mathrm{III}}\right] 5007$ FWHM, "1": the fitting result is reliable. "2": the FWHM can not be well fitted due to the low quality of the spectra or suffer serious absorptions around the emission lines. 